\title{
Uncertainty Study of Reflectance Measurements for Concentrating Solar Reflectors
}

\author{
Francisco Buendía-Martínez ${ }^{\circledR}$, Aránzazu Fernández-García ${ }^{(}$, Florian Sutter $^{(}$, Lucía Martínez-Arcos $^{(}$, \\ Tomás Jesús Reche-Navarro ${ }^{\circledR}$, Alejandro García-Segura ${ }^{\circledR}$, and Loreto Valenzuela ${ }^{\circledR}$
}

\begin{abstract}
The solar reflector is one of the main components of concentrated solar thermal systems. Therefore, accurate knowledge of its solar-weighted, near-specular reflectance is highly important. Currently, this parameter cannot be properly measured with a single commercial instrument. There is a great interest in having a suitable procedure that can guarantee the accuracy of reflector quality analysis, which already led to the publication of an international measurement guideline (title "Parameters and method to evaluate reflectance properties of reflector materials for concentrating solar power technology"). Still, more research work is needed to improve the state of the art. At present, both the specular reflectance and the spectral hemispherical reflectance are measured by using commercial portable reflectometers and spectrophotometers, respectively, to gain enough information. This article concentrates on the evaluation and calculation of the type-B (nonstatistical) uncertainties associated with these employed instruments and, therefore, leads to a more accurate definition of the measurement uncertainty. Considering type-B uncertainty, the expanded uncertainties of measurements for most of the reflector types are $U_{\mathrm{B} \text {,ref }}=\mathbf{0 . 0 0 6}$ for monochromatic specular reflectance and $U_{\mathrm{B} \text {,spec }}=0.016$ for solar-weighted hemispherical reflectance.
\end{abstract}

Index Terms-Accurate measurement, concentrating solar thermal, instrument uncertainty, monochromatic specular reflectance, solar reflector, spectral hemispherical reflectance.

\section{INTRODUCTION}

G LOBAL warming, worldwide fossil fuel shortage in the near future and restrictions on carbon emissions are increasing the importance of renewable energy resources. The Sun radiates more energy in one second than the whole amount of energy used since the beginning of humanity [1]. The main technologies that harvest solar energy are photovoltaics and concentrated solar thermal (CST) systems. On the one

Manuscript received October 31, 2019; revised January 9, 2020; accepted January 28, 2020. Date of publication February 24, 2020; date of current version August 11, 2020. This work is part of the project RAISELIFE that has received funding from the European Union's Horizon 2020 Research and Innovation Programme under grant agreement No 686008. The Associate Editor coordinating the review process was Datong Liu. (Francisco Buendia-Martínez, Florian Sutter, Lucía Martínez-Arcos, Tomás Jesús Reche-Navarro, Alejandro García-Segura, and Loreto Valenzuela contributed equally to this work.) (Corresponding author: Aránzazu Fernández-García.)

Francisco Buendía-Martínez, Aránzazu Fernández-García, Lucía Martínez-Arcos, Alejandro García-Segura, and Loreto Valenzuela are with CIEMAT-Plataforma Solar de Almería, 04200 Almería, Spain (e-mail: arantxa.fernandez@psa.es).

Florian Sutter and Tomás Jesús Reche-Navarro are with the German Aerospace Center (DLR), 04200 Almería, Spain.

Color versions of one or more of the figures in this article are available online at http://ieeexplore.ieee.org.

Digital Object Identifier 10.1109/TIM.2020.2975387 hand, photovoltaic energy uses solar cells to directly generate electricity. This energy has been very common in electricity production for many years [2]. On the other hand, CST technology has been under investigation for more than a century, but it has been commercialized in the last four decades [3]. CST technology has been delayed in market development since the 1980s because of market resistance to large plant sizes and poor political and financial support from incentive programs [4]. In the last decade, public programs in several countries around the world (led by Spain and USA) have promoted a rapid growth in both the basic technology and the market establishment [5].

All CST systems, from line focusing to point focusing ones, are based on large areas covered by solar concentrators (i.e., reflecting mirrors with the proper shape) that concentrate the direct solar radiation into a receiver where a circulating fluid increases its enthalpy. The deployment of this technology is linked to the development of cost-effective components, which, in the case of the optical concentrator, means durable reflectors with high solar-weighted near-specular reflectance. Consequently, the proper assessment of this optical parameter is a crucial issue that is receiving special attention from the international solar community [6].

Research work of the last few years regarding the reflectance measurement procedure [7]-[11] and instruments [12]-[16] of solar mirror materials has advanced as far as the publication of a reflectance measurement guideline within the SolarPACES Task III [17]. This procedure is already established as the standard protocol to be followed by the official norms of solar reflectors [18]. According to this guideline and due to the lack of appropriate measurement equipment [19], the evaluation of nonhighly specular mirrors (such as aluminum or polymer film ones), as well as aged and soiled mirrors, must consider the results obtained separately from instruments such as specular reflectometers and spectrophotometers. In addition, a proper optical characterization of solar reflectors involves not only defining a precise measurement protocol to achieve a correct real value but also providing the specific and detailed uncertainty of the measurement instruments.

This article is focused on a thorough study on the evaluation and calculation of the type-B (nonstatistical) uncertainties associated with the commercial instruments typically employed and, thus, leads to a more accurate and deep definition of the reflectance uncertainty. Moreover, the goal of this article is to provide proof and clarification about certain 
details of the reflectance measurement procedure and offer suggestions on how to enable higher accuracy. This study was conducted with the most commonly used reflectance measurement devices, that is, a Perkin Elmer (PE) Lambda 1050 spectrophotometer and a 15R-USB Devices and Services (D\&S) portable reflectometer.

\section{Methods}

This section describes a frequently employed method to measure the reflectance of concentrating solar reflectors, the instruments and samples used in this study, and the methodology followed to verify the measurement procedure and calculate its uncertainty. Nomenclature applied is according to [18] and [20].

\section{A. Reflectance Definitions}

The most precise way to characterize a reflector material for CST applications is to measure its specular reflectance, $\rho_{\lambda, \varphi}\left(\lambda, \theta_{i}, \varphi\right)$, as a function of the wavelength, $\lambda$, the incidence angle, $\theta_{i}$, and the acceptance angle, $\varphi$, in a proper range [21]. It is not possible to directly obtain this parameter with a unique instrument at the current state of the art. As a compromise solution, the required information to optically characterize solar reflectors (mainly in nonspecular, aged, and soiled mirrors) is typically obtained by using two different commercial instruments [7], [17].

Spectral reflectance is generally obtained with a commercial spectrophotometer that measures the hemispherical reflectance, thus covering the ultraviolet (UV), visible (Vis), and near-infrared (NIR) ranges. This means that all light reflected in the hemisphere is measured regardless of its directionality. This optical parameter is the spectral hemispherical reflectance, $\rho_{\lambda, h}\left(\lambda, \theta_{i}, h\right)$, which depends on $\lambda$ and $\theta_{i}$, where, in this case, $\varphi$ is denoted by $h$ to indicate the complete hemisphere. Hemispherical spectrum is weighted with the solar spectrum to obtain the solar-weighted value in a specific $\lambda$ range, $\rho_{s, h}\left(\left[\lambda_{a}, \lambda_{b}\right], \theta_{i}, h\right)$ [22]. This parameter allows assessing the optical behavior in the solar spectrum but presents the disadvantage of missing the required information about its specular performance.

Since solar concentrators only use the reflected radiation in the near-specular direction, their performance highly depends on the amount of beam spread. Therefore, the solar specular reflectance, $\rho_{s, \varphi}\left(\left[\lambda_{a}, \lambda_{b}\right], \theta_{i}, \varphi\right)$, must also be characterized. This value describes the amount of energy reflected around the specular direction according to the law of reflection and is bounded by $\varphi$. Measurement of spectral specular reflectance is problematic at this time because adequate instrumentation that can define representative $\varphi$ for CST technologies and in a wide $\lambda$ range is not marketed although, in the past years, several prototype instruments and methods have been developed to determine it [10], [13]-[15]. In commercial reflectometers, specular reflectance can only be appropriately measured at a certain selected $\lambda$ and a fixed near-normal $\varphi$. The value supplied is the monochromatic specular reflectance, $\rho_{\lambda, \varphi}\left(\lambda, \theta_{i}, \varphi\right)$, which gives useful information about the specular behavior but is insufficient because only a small $\lambda$ range is registered.

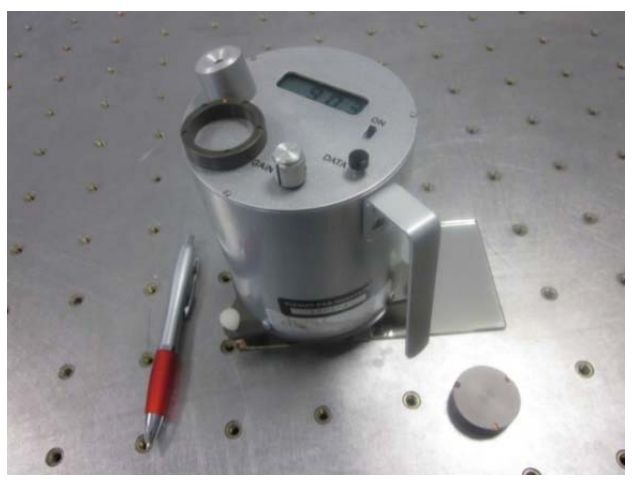

Fig. 1. Portable specular reflectometer 15R-USB by D\&S.

TABLE I

MAIN CHARACTERISTICS OF THE REFLECTOMETER AND SPECTROPHOTOMETER

\begin{tabular}{|c|c|c|}
\hline Equipment & Reflectometer & Spectrophotometer \\
\hline Manufacturer & Devices and Services Co. & Perkin Elmer \\
\hline Model & $15 \mathrm{R}$-USB & Lambda 1050 \\
\hline Measurement principle & A source lamp and a detector & A source lamp and an integrating sphere \\
\hline Type of measurements & $\begin{array}{l}\text { Monochromatic specular } \\
\text { reflectance at selected } \varphi\end{array}$ & Spectral hemispherical reflectance \\
\hline Light source & LED & Halogen and deuterium lamps \\
\hline Detector & Silicon cell & $\begin{array}{l}\text { Photomultiplier (UV/Vis), Peltier controlled } \\
\qquad \mathrm{PbS} \text { and InGaAs (NIR) }\end{array}$ \\
\hline Incidence angle, $\theta_{i}\left({ }^{\circ}\right)$ & 15 & 8 \\
\hline Beam spot size $(\mathrm{mm})$ & 10 (diameter) & $17 \times 9$ \\
\hline Wavelength range $(\mathrm{nm})$ & $635-685 \mathrm{~nm}$, peak at $660 \mathrm{~nm}$ & {$[320,2500]$} \\
\hline Acceptance angle, $\varphi$ (mrad) & $3.5,7.5,12.5,23.0$ & Hemisphere \\
\hline Accuracy $(-)$ & \pm 0.002 (at $660 \mathrm{~nm})$ & \pm 0.007 (at $635 \mathrm{~nm})$ \\
\hline Resolution (-) & \pm 0.001 & See Section 3.2.3 \\
\hline Adaptable to mirror curvature & Yes & No \\
\hline Suitable for $2^{\circ}$ surface mirrors & Yes & Yes \\
\hline Influence of external light & No (chopped light source) & No (sample port protected) \\
\hline Battery autonomy (h) & $25-30$ & No applicable, lab equipment \\
\hline Weight $(\mathrm{kg})$ & 1.1 & 80.0 \\
\hline Operating temperature $\left({ }^{\circ} \mathrm{C}\right)$ & $0-50$ & $10-35$ \\
\hline References & {$[24,25]$} & {$[26,27]$} \\
\hline
\end{tabular}

\section{B. Measurement Devices}

This section presents a description of the measurement devices analyzed in this work. In both cases, the two specific instruments selected were chosen because they are the most commonly used at present [19], and to the best of the authors' knowledge, they are the most appropriate for measuring the reflectance of solar reflectors.

1) Reflectometer: The 15R-USB by D\&S Co. was selected to measure $\rho_{\lambda, \varphi}$ (see Fig. 1). This portable reflectometer was developed in cooperation with the Sandia National Laboratories [23] with the specific purpose of measuring reflectors for CST systems. Table I presents the main features of this device.

The reasons for choosing this equipment are that the specular reflectance is directly measured by choosing $\varphi$ in the appropriate range; it allows for the adjustment of the beam path, so that the first and second surfaces or curved mirrors can 


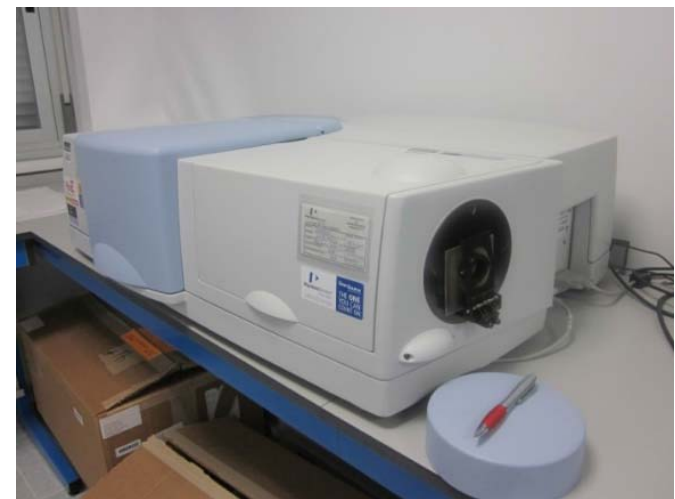

Fig. 2. Spectrophotometer Lambda 1050 by PE.

TABLE II

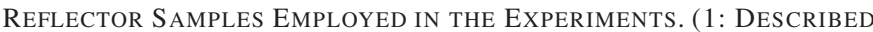
In [37] AS MATERIAL A AND 2: DESCRIBED IN [37] AS MATERIAL G)

\begin{tabular}{|c|c|c|c|}
\hline Sample ID & Type & Size $\left(\mathrm{mm}^{2}\right)$ & Description \\
\hline Silvered-glass $0.95 \mathrm{~mm}$ & $2^{\text {nd }}$ surface & $100 \times 100$ & Thin low-iron silvered glass of $0.95 \mathrm{~mm}$ thickness \\
\hline Silvered-glass $2.1 \mathrm{~mm}$ & $2^{\text {nd }}$ surface & $100 \times 100$ & Thick low-iron silvered glass of $2.1 \mathrm{~mm}$ thickness \\
\hline Silvered-glass $4.0 \mathrm{~mm}$ & $2^{\text {nd }}$ surface & $100 \times 100$ & Thick low-iron silvered glass of $4 \mathrm{~mm}$ thickness \\
\hline Silvered polymer film \#1 & $1^{\text {st }}$ surface & $70 \times 70$ & $\begin{array}{l}\text { Polymer film from manufacturer A, without degradation. } \\
\text { Sample glued to } 4 \mathrm{~mm} \text { glass substrate }\end{array}$ \\
\hline Silvered polymer film \#2 & $1^{\text {st }}$ surface & $70 \times 70$ & $\begin{array}{l}\text { Polymer film from manufacturer B, with low degradation. } \\
\text { Sample glued to } 4 \mathrm{~mm} \text { glass substrate }\end{array}$ \\
\hline Aluminum \#1 & $1^{\text {st }}$ surface & $100 \times 100$ & $\begin{array}{l}\text { Aluminum with } 2 \text { physical vapor deposition layers }\left(\mathrm{TiO}_{2}\right. \\
\left.\text { and } \mathrm{SiO}_{2}\right) \text { and special lacquer on top for outdoor }\end{array}$ \\
\hline Aluminum $\# 2$ & $1^{\text {st }}$ surface & $100 \times 100$ & Aluminum with special lacquer on top for outdoor ${ }^{2}$ \\
\hline
\end{tabular}

be measured; and it has no influence on external stray light and it is suitable for field measurements. Although the instrument comes with a reference mirror of known reflectance (see bottom right of Fig. 1), which can be inserted in a fixed position for calibration, an external calibration is recommended [19]. The instrument produces a collimated beam to a diameter of $10 \mathrm{~mm}$, so that all of the reflected beam can be collected by the 22-mm-diameter receiver lens. This device has been extensively employed in research activities by a number of institutions [28]-[32].

2) Spectrophotometer: $\rho_{\lambda, h}$ was measured with a Lambda 1050 two-beam scanning spectrophotometer by PE, equipped with an integrating sphere accessory of $150 \mathrm{~mm}$ diameter (see Fig. 2). In general, this PE device is frequently used for a wide range of applications to measure the transmittance, the absorptance, and the reflectance of solutions and opaque materials and has been extensively employed to characterize solar reflectors [33]-[35]. Table I presents the main features of this device. Measurements were taken at a 5-nm step.

\section{Sample Description}

Several solar reflector types are employed in CST applications. They can be classified as silvered-glass reflectors, aluminum reflectors, and silvered-polymer films [36]. All types of solar reflectors were included in this article. Tables II and Fig. 3 show the sample identification (ID) and a brief description of the samples used as measurement samples in

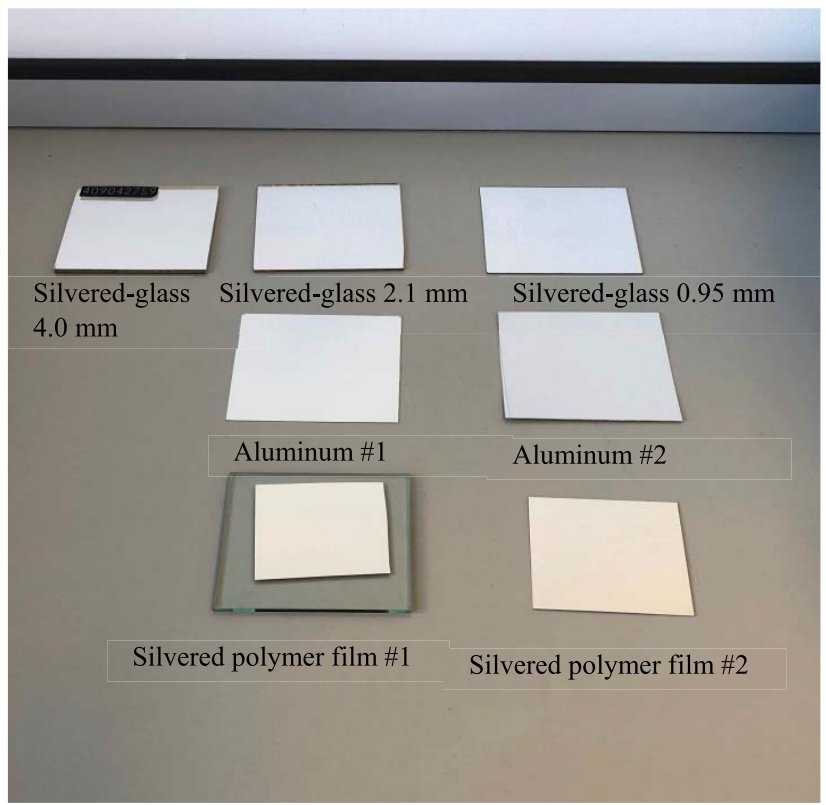

Fig. 3. Reflector types studied in this article.

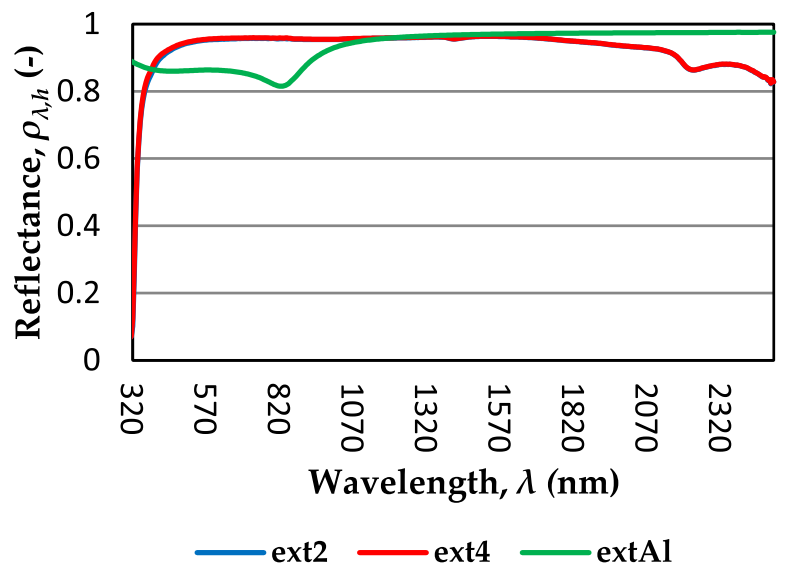

Fig. 4. Calibrated hemispherical reflectance spectra of reference standards.

the tests. Polymer films were applied to a glass substrate to give them enough rigidity.

Table III presents the main features of the coupons used as reference standards, as given by the manufacturers who also provided the calibration data. The three standard mirrors listed in Table III are named external because they can be used for all the instruments. However, there are other kinds of reference mirrors labeled as internal mirrors, which are provided by the manufacturer $(D \& S)$ for each specific reflectometer. The uncertainty data do not include the coverage factor (see Section II-D).

Finally, Fig. 4 shows the calibrated hemispherical spectra of all reference standards described in Table III. As can be observed, the highest reflectance values are reached by the two glass references, and the aluminum spectrum is considerably lower in the visible range.

\section{Uncertainty Calculation}

The process to calculate uncertainties associated with experimentally measured magnitudes was done by following the international "Guide to the Expression of Uncertainty 
TABLE III

REFERENCE STANDARDS EMPLOYED IN THE EXPERIMENTS

\begin{tabular}{|c|c|c|c|c|c|}
\hline Sample ID & Company & Size $\left(\mathrm{mm}^{2}\right)$ & Serial Number & Uncertainty & Description \\
\hline ext2 & $\begin{array}{c}\text { OMT } \\
\text { Solutions }\end{array}$ & $100 \times 100$ & OMT-216035-01 & 0.0015 & $\begin{array}{l}\text { Thick low-iron silvered-glass mirror } \\
\text { of } 2 \mathrm{~mm} \text { thickness }\end{array}$ \\
\hline ext4 & $\begin{array}{c}\text { OMT } \\
\text { Solutions }\end{array}$ & $100 \times 100$ & OMT-214044-02 & 0.0015 & $\begin{array}{l}\text { Thick low-iron silvered-glass mirror } \\
\text { of } 4 \mathrm{~mm} \text { thickness }\end{array}$ \\
\hline extAl & NRC & $70(\varnothing)$ & PAV-D-2 & 0.0013 & $\begin{array}{l}1^{\text {st }} \text { surface aluminum coat on top of } \\
\text { high quality glass substrate }\end{array}$ \\
\hline
\end{tabular}

in Measurement" [38]. The reflectance uncertainties refer to the specular and hemispherical reflectance measurements performed with the corresponding instruments. The uncertainty of the monochromatic specular reflectance, $u_{\rho_{\lambda, \varphi}\left(\lambda, \theta_{i}, \varphi\right)}$, is obtained by considering type-A and type-B uncertainties of the reflectometer, $u_{\text {refl }}$

$$
u_{\rho_{\lambda, \varphi}\left(\lambda, \theta_{i}, \varphi\right)}=u_{\mathrm{refl}}=\sqrt{u_{\mathrm{A}, \mathrm{refl}}^{2}+u_{\mathrm{B}, \mathrm{refl}}^{2}} .
$$

On the other hand, the uncertainty of the spectral or solar hemispherical reflectance, $u_{\rho_{\lambda, h}\left(\lambda, \theta_{i}, h\right)}$, measured with the spectrophotometer, $u_{\text {spec }}$, is calculated as follows:

$$
u_{\rho_{\lambda, h}\left(\lambda, \theta_{i}, h\right)}=u_{\mathrm{spec}}=\sqrt{u_{\mathrm{A}, \mathrm{spec}}^{2}+u_{\mathrm{B}, \mathrm{spec}}^{2}}
$$

where $u_{\mathrm{A} \text {,refl }}$ and $u_{\mathrm{A} \text {,spec }}$ are the objective uncertainty obtained from the statistical analysis of series of observations, and $u_{\mathrm{B}, \text { refl }}$ and $u_{\mathrm{B} \text {,spec }}$ have a subjected character and they are calculated by means of other than the statistical analysis of series of observations. Therefore, the purpose of the type$\mathrm{A}$ and type-B classifications is to indicate the two different ways of evaluating uncertainty components and for convenience of discussion only; the classification is not meant to indicate that there is any difference in the nature of the components resulting from the two types of evaluation [38]. Both of them are based on probability distributions (Gaussian, rectangular, triangular, and trapezoidal). The calculation of the uncertainty linked to a set of observations depends on the probability distribution, being the most common one, i.e., the Gaussian or normal distribution whose uncertainty is the standard deviation [38]. The normality of the distributions was checked by using the contrast of the hypothesis through the Shapiro-Wilk, ANOVA, and Wilcoxon tests. The type-B uncertainty of the measurement instruments was derived from the available information given by the manufacturers and performing statistical observations through specific experiments of those key factors whose influence is missing. Ten repetitions in each experiment with both instruments were performed.

\section{E. Type-B Uncertainty of the Reflectometer}

The uncertainties considered relevant in the $\rho_{\lambda, \varphi}$ measurement process with the reflectometer are given as follows:

1) accuracy, $u_{\text {refl,acc }}$ (given by the manufacturer as reproducibility, Table I);

2) resolution, $u_{\text {reff, res }}$ (given by the manufacturer, Table I);

3) calibration quality of the reference mirror, $u_{\text {refl,cal }}$ (given by the manufacturer, Table III);

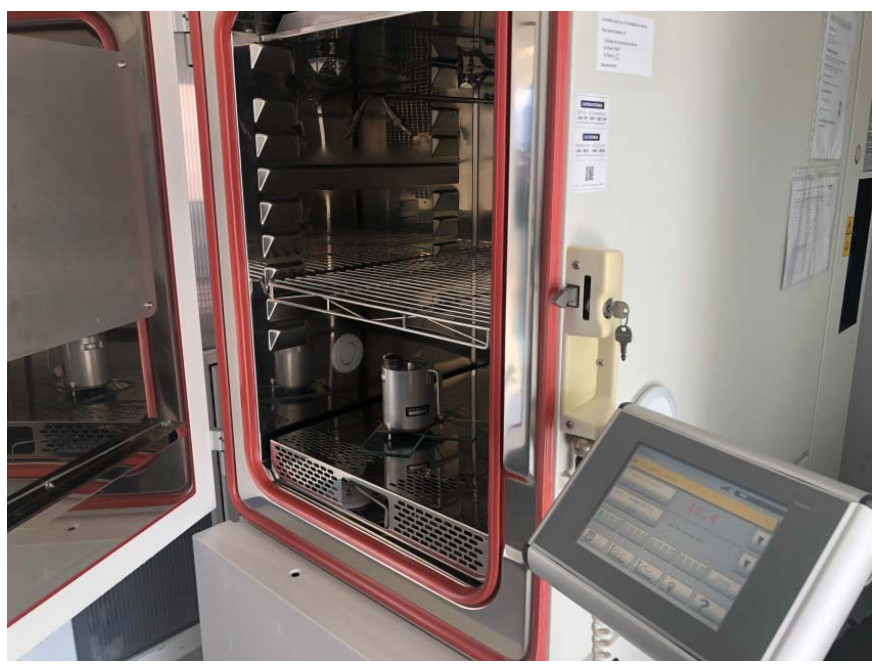

Fig. 5. Ambient temperature test carried out in a weathering chamber.

4) influence of the ambient temperature, $u_{\text {reff,tem; }}$;

5) influence of the reflectometer unit itself, $u_{\text {refl, unit }}$;

6) influence of the reference mirror (external or internal), $u_{\text {refl, ref }}$

7) stability over time, $u_{\text {refl,time }}$;

8) influence of the ambient light, $u_{\text {refl,light }}$;

9) influence of the acceptance angle, $u_{\mathrm{refl}, \varphi}$;

10) influence of the reflectometer's central screw position, $u_{\text {refl, scr }}$

11) influence of the operator, $u_{\text {reff,ope }}$;

12) influence of the curvature, $u_{\text {refl,curv }}$.

As there is not any known relationship between the abovementioned uncertainties, the type-B uncertainty of the reflectometer, $u_{\mathrm{B}, \text { refl }}$, can be calculated as follows:

$$
u_{\mathrm{B}, \text { refl }}=\sqrt{\begin{array}{l}
u_{\text {refl,acc }}^{2}+u_{\text {refl,cal }}^{2}+u_{\text {refl, res }}^{2}+u_{\text {refl,tem }}^{2}+u_{\text {refl,unit }}^{2} \\
+u_{\text {refl,ref }}^{2}+u_{\text {refl,time }}^{2}+u_{\text {refl,light }}^{2} \\
+u_{\text {refl, } \varphi}^{2}+u_{\text {refl,scr }}^{2}+u_{\text {refl,ope }}^{2}+u_{\text {refl,curv }}^{2}
\end{array}}
$$

Only the uncertainties associated with the first three influences are known. The following sections include a detailed description of all the related tests performed to calculate the influence of the other parameters and the corresponding uncertainties when it is necessary.

1) Ambient Temperature: Although the reflectance is an optical parameter influenced by the temperature [39], according to the instrument manufacturer, any temperature effect is internally minimized [25]. An experiment was performed to verify it, which consisted in measuring all the mirror samples, ten times every $5^{\circ} \mathrm{C}$ within the operating temperature range, $T=[10,45]{ }^{\circ} \mathrm{C}$. The test was performed inside a weathering chamber model SC 340 manufactured by ATLAS (see Fig. 5). For each temperature step, the mirror sample stood inside the chamber together with the reflectometer for $30 \mathrm{~min}$ before the measurement. The experiment was done by calibrating the instrument only at the standard ambient temperature $\left(T=22.5^{\circ} \mathrm{C}\right)$ as well as performing intermediate recalibrations at each temperature step. In the rest of the tests, ambient temperature $=22.5^{\circ} \mathrm{C}$ was kept. 


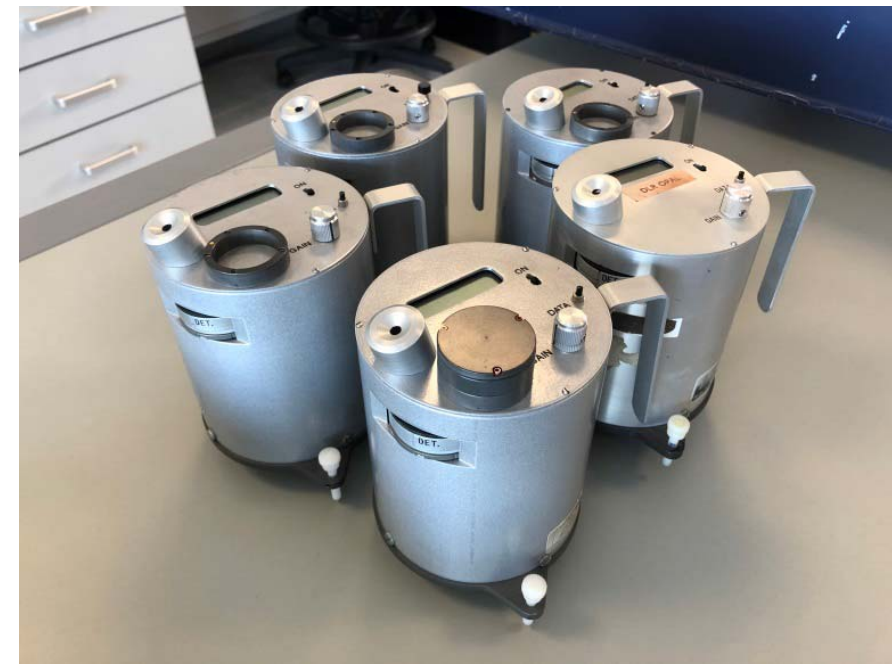

Fig. 6. Different reflectometers used for this experiment.

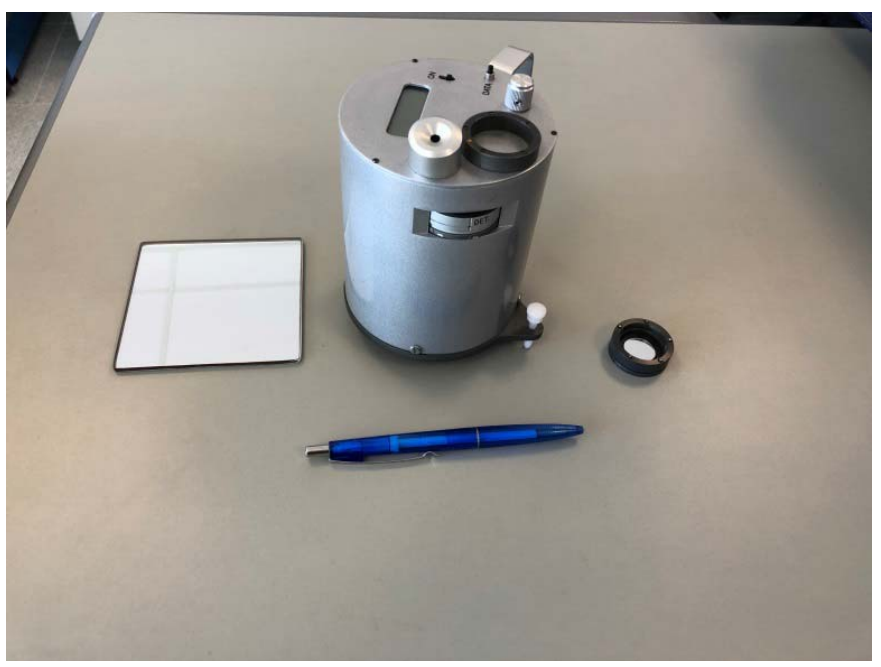

Fig. 7. Different reference mirrors used for this experiment: ext4 (left) and internal reference mirror (right).

2) Reflectometer Unit Itself: This test is focused on the calculation of the uncertainty derived for the use of one specific instrument. It was performed with five different D\&S reflectometers with serial numbers $060,110,116,117$, and 119 (see Fig. 6). To avoid any influence of the sample behavior, the most stable and homogeneous sample (4.0-mm silveredglass) was employed in the measurements (ten repetitions with each unit), and the results obtained are considered valid for the rest of the materials. The device with serial number 117 was chosen for the rest of the tests.

3) Reference Mirror: The aim of this test was to calculate the uncertainty associated with the calibration mirror by comparing the internal one with the 4.0-mm silvered-glass external reference mirror (ext4) (see Fig. 7). To avoid any influence of the sample behavior, the most stable and homogeneous sample (4-mm silvered-glass) was measured (ten times with each reference mirror), and the results achieved are valid for the rest of the materials. The reflectometers were calibrated with this external reference mirror in the rest of the testing campaign.

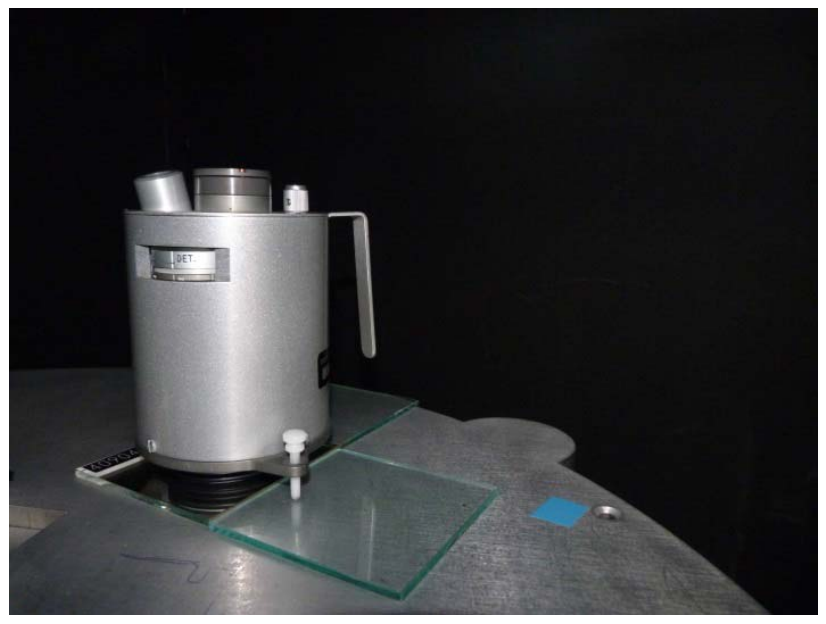

Fig. 8. Ambient light test performed under dark conditions.

4) Stability Over Time: This test was performed to check the stability of the instrument over time while keeping the rest of the operating conditions constant. The 4-mm silvered-glass sample was measured ten times during a full working day (i.e., 7 h).

5) Ambient Light: According to the reflectometer's manufacturer, the light source is chopped electronically at a rate of about $90 \mathrm{~Hz}$, so that the stray light will not cause measurement errors [24]. A test was conducted to check the validity of this system. The 4.0-mm silvered-glass sample was measured ten times in an illuminated room (ambient light) and another ten times in dark conditions by covering the instrument and the operator with an opaque fabric (see Fig. 8). The rest of the tests were performed with ambient light.

6) Acceptance Angle: The operator of the D\&S reflectometer can select one out of three apertures that define $\varphi$ in the path of the reflected beam by rotating the thumbwheel on the side of the instrument [25]. A test was performed to evaluate the influence of $\varphi$ on the reflectance of all samples because it is well-known that the scattering phenomenon depends on the material type [14]. Different $\varphi$ used were $\varphi=\{7.5,12.5,23.0\}$ mrad (ten repetitions were done with each $\varphi$ ). The $\varphi$ selected in the rest of the study was $12.5 \mathrm{mrad}$ because it is the one recommended for parabolic-trough collectors [7].

7) Central Screw's Position: The D\&S reflectometer has a central screw that must be adjusted depending on the frontlayer thickness. The length of the central screw controls the distance of the incidence beam path before reaching the reflective surface of the sample. According to the manufacturer instructions [25], for the first-surface mirrors, it has to be screwed to the maximum extended position. For the secondsurface mirrors, it should be adjusted by an amount equal to the thickness of the mirror divided by the index of refraction of the material. Assuming that the index of the refraction of glass is 1.5 , the adjustment is about 1 turn for each $1.27 \mathrm{~mm}$ thickness. An experiment was done to verify if modifications of the central screw position cause variations in the reflectance. The reflectance of three mirror samples with different frontlayer thicknesses (aluminum \#1-first surface and 0.95-mm silvered-glass and 4.0-mm silvered-glass—-second surface) 

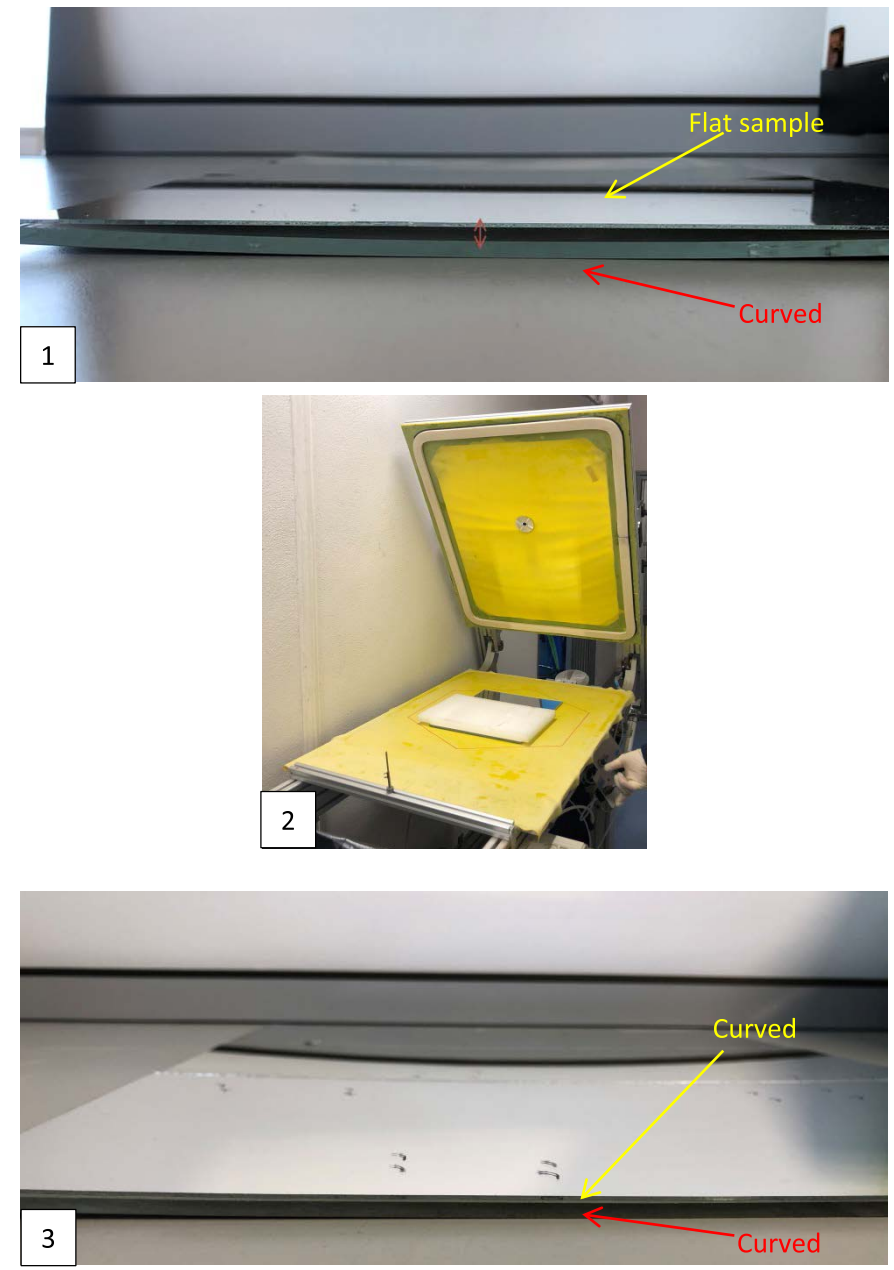

Fig. 9. Process to change the curvature: 1) flat sample and curved samples; 2) curvature of the flat sample is changed by a bending machine; and 3) original flat sample achieved the same curvature as that of the real PTC facet.

was checked at ten positions of the central screw, including the corresponding turn according to the mirror thickness. The results obtained are considered valid for the other samples with similar front-layer thickness. The rest of the tests were performed with the central screw in a fixed position.

8) Operator: This test was performed to calculate the uncertainty linked to the experience of the operator. The samples were measured by three different operators with high, medium, and low experience (ten repetitions with each operator). In this case, all the samples were measured because the difficulty of the calibration process depends on the material type. The rest of the studies were carried out by the expert operator.

9) Curvature of the Mirror: The influence of the mirror curvature was studied with three different flat solar mirrors (see Table II): 0.95-mm silvered-glass, silvered polymer film $\# 1$, and aluminum \#2. A bending machine was used to modify the shape of the flat samples, thus providing the same curvature as a real parabolic-through collector (PTC) facet. To do that, the samples were glued to a real PTC facet piece $\left(30 \times 40 \mathrm{~cm}^{2}\right)$ and submitted to a vacuum atmosphere (see Fig. 9). In this case, the selected mirror sample size was $30 \times 13 \mathrm{~cm}^{2}$ to have enough size to copy the curvature of a real facet. Every sample was measured in the same spot both in the flat and curved statuses. The comparison of the results for the flat and curved shapes will give the possible influence of the curvature. To have enough statistical information, this measurement was repeated ten times.

\section{F. Type-B Uncertainty of the Spectrophotometer}

$\rho_{\lambda, h}$ measurements done with the spectrophotometer also depend on several nonstatistical uncertainties. For this article, the type- $\mathrm{B}$ uncertainties considered relevant in the whole reflectance measurement process with the spectrophotometer are given as follows:

1) accuracy, $u_{\text {spec }}$,acc (given by the manufacturer, Table I);

2) resolution, $u_{\text {spec,res }}$;

3) calibration quality of the reference mirror, $u_{\text {spec }}$,cal (given by manufacturer, Table III);

4) influence of the ambient temperature, $u_{\text {spec }, \text { tem }}$;

5) influence of the spectrophotometer unit itself, $u_{\text {spec,unit }}$;

6) influence of the reference mirror (external), $u_{\text {spec,ref }}$;

7) stability over time, $u_{\text {spec, time }}$;

8) influence of the ambient light, $u_{\text {spec,light }}$;

9) influence of the detector response time, $u_{\text {spec,det }}$;

10) influence of the curvature, $u_{\text {spec,curv }}$.

There is not any known relationship between the abovementioned uncertainties. Therefore, the combined type-B uncertainty of the spectrophotometer, $u_{\mathrm{B}, \mathrm{spec}}$, can be calculated by the following equation:

$$
u_{\mathrm{B}, \mathrm{spec}}=\sqrt{\begin{array}{l}
u_{\text {spec,acc }}^{2}+u_{\text {spec, cal }}^{2}+u_{\text {spec, res }}^{2}+u_{\text {spec,tem }}^{2}+u_{\text {spec, unit }}^{2} \\
+u_{\text {spec,ref }}^{2}+u_{\text {spec, time }}^{2}+u_{\text {spec, light }}^{2} \\
+u_{\text {spec, det }}^{2}+u_{\text {spec,curv }}^{2} .
\end{array}}
$$

The following sections include a detailed description of all the tests performed to evaluate the unknown uncertainties of the spectrophotometer (i.e., all the previously listed except the first two).

1) Ambient Temperature: According to [17], the temperature is a factor that could significantly change the accuracy of the spectrophotometer. To check this influence, the device was situated in a room where the temperature was controlled by a thermostat and verified by a thermometer. All samples were measured ten times at $T=\{16,26\}{ }^{\circ} \mathrm{C}$. These minimum and maximum ambient temperatures were chosen according to the usual working temperature conditions in a laboratory. In the rest of the tests, $T=22.5^{\circ} \mathrm{C}$ was kept.

2) Spectrophotometer Unit Itself: In a round Robin test that was performed in an earlier research work in 2010 [7], a set of samples similar to the selected ones in this study were evaluated using the spectrophotometer of this study and a Lambda 950 by PE at the DLR Quarz Laboratory, Cologne, Germany, keeping the rest of the measurement conditions constant. This gives hints of the uncertainty concerning different instruments at different laboratories. The results obtained a normal distribution with a standard deviation of $\sigma \leq 0.002$. Therefore, an uncertainty of $u_{\text {spec, unit }}=0.002$ is considered in this study. It indicates that this kind of measurement can be performed very stable if a good calibration of the reference mirror is ensured and the same method is applied. However, 
it is advisable to always use the same spectrophotometer because in this case, the uncertainty would be negligible. In this article, all the experiments were performed with the same spectrophotometer.

3) Reference Mirror: It is pointed out in the literature [40], [41] that the reference sample for measurements with an integrating sphere should have the same properties as the test sample to be measured to acquire accurate results. This refers to its specularity, the grade of reflectance, and also if it is a first or second surface mirror. The purchase of several kinds of calibrated reference mirrors may be expensive, and it is also hindered by the lack of products in the market. It would be easier and cheaper to settle on only one stable reference mirror that can be used for all types of test samples.

To find out if this can be realized without compromising the accuracy, a set of tests was performed with two representative samples, aluminum \#1 and 4-mm silvered-glass samples. Each sample was measured with all the three reference mirrors listed in Table III. For the rest of the experiment, the reference mirror selected was the 4-mm silvered-glass (ext4).

4) Stability Over Time: The SolarPACES Reflectance Guideline suggests that the stability over time is a parameter that could affect the spectrophotometer uncertainty [17]. Therefore, a test was carried out to assess this influence. In this case, the 4-mm silvered-glass sample was measured (to avoid any influence of the material itself) during $14 \mathrm{~h}$ at every $3 \mathrm{~min}$ (i.e., 280 times) while keeping the rest of the measurement conditions constant.

5) Ambient Light: After the years of experience, it has been observed that the external light has a major influence on the reliability of a measurement with the spectrophotometer. For this reason, a test was performed to assess the importance of controlling this parameter. A homemade cover of an opaque plastic was manufactured by OPAC operators. The spectrophotometer was totally covered with this gadget, and ten measurements of the $4.0-\mathrm{mm}$ silvered-glass samples were done with and without the cover. For the rest of the tests, a cover was used.

6) Detector Response Time: Another important factor observed in the laboratory that could affect the result is the detector response time, which can be varied in a wide range. Actually, it can be changed both within a wavelength range and across the whole spectrum. In this test, the reflectance variability was studied when the spectrophotometer worked with a detector response time of 1 and $0.04 \mathrm{~s}$, which are the slowest and fastest, respectively. In addition, a mix of these two detector response times was checked. Ten measurements were performed for each response time with the 4-mm silvered-glass sample. It should be taken into account that at $1 \mathrm{~s}$, the measurements involved around $10 \mathrm{~min}$, four times more than at $0.04 \mathrm{~s}$. For the rest of the tests, a combination of detector response times was selected, according to the results obtained (see Section III-B6).

7) Curvature of the Mirror: In this article, reflectance measurements were taken in the same spot for the flat and curved mirror samples in order to verify the uncertainty that could provoke the curvature, as it was previously explained in Section II-E9.

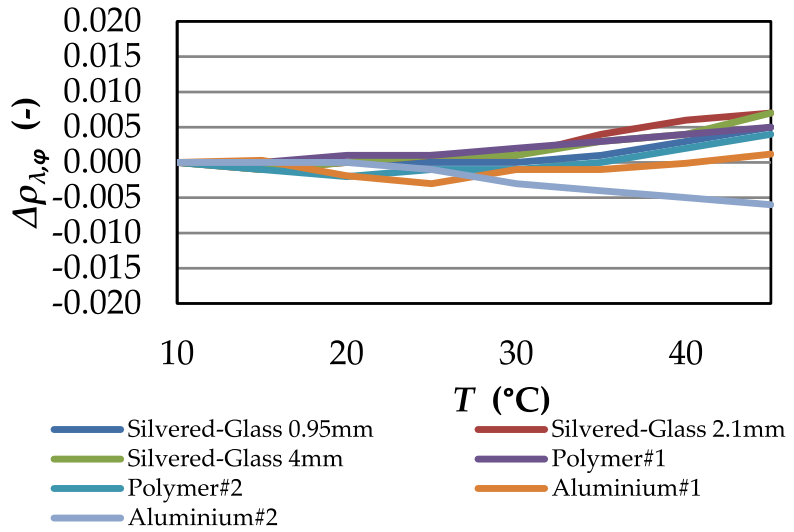

Fig. 10. Influence of the ambient temperature in the reflectance for all reflector samples without intermediate calibrations.

\section{RESULTS}

This section includes the results obtained from all the tests performed to calculate the unknown type-B uncertainties associated with the reflectometer and the spectrophotometer measurements.

\section{A. Type-B Uncertainties of the Reflectometer}

Results of the tests described in Section II-E to calculate the type-B uncertainties of the reflectometer are presented in this section.

1) Ambient Temperature: In general, differences due to the temperature changes are not considered as an uncertainty but considered as a measurement correction. This behavior depends on the reflector materials because their chemical structure may be affected by thermal expansion. The results of the ambient temperature study are shown in Fig. 10, where the reflectance differences, $\Delta \rho_{\lambda, \varphi}$, with respect to the value at the lowest temperature $\left(T=10{ }^{\circ} \mathrm{C}\right)$ are presented, for all reflector samples.

As shown in Fig. 10, temperature variations affect the reflectance when the calibration is performed only once. The graph shows that the measured reflectance of silvered-glass and polymer samples increases when ambient temperature increases, mainly for values above $25^{\circ} \mathrm{C}$. However, the measured reflectance in the aluminum \#2 sample is totally opposite to the rest of the samples because it decreases when the temperature increases. Finally, the measured reflectance of the aluminum \#1 sample does not show a clear tendency.

On the contrary, when intermediate calibration is carried out in every temperature step (see Fig. 11), no influence on the reflectance was observed for silvered materials (both silvered-glass and polymer film reflectors). However, in the case of aluminum\#1 and \#2 samples, it was noticed that their reflectance decreases when the temperature rises. The change in the measured reflectance between $10{ }^{\circ} \mathrm{C}$ and $45{ }^{\circ} \mathrm{C}$ is very drastic in aluminum materials, thus reaching values up to $-0.0068 \mathrm{ppt}$ for aluminum \#1 and $-0.017 \mathrm{ppt}$ for aluminum \#2. This change is originated by a change in sample flatness, which can strongly affect specularity (the mirror 


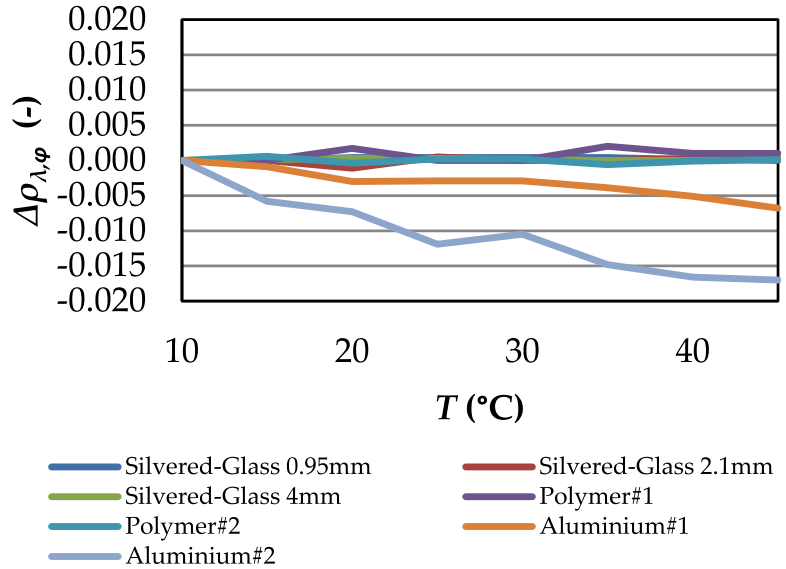

Fig. 11. Influence of the ambient temperature in the reflectance for all reflector samples with intermediate calibrations.

thickness is only $0.5 \mathrm{~mm}$ ). The changes in sample flatness cannot be compensated by the calibration mirror.

Taking into consideration the results achieved in the two tests performed, it is concluded that the influence of the temperature on the reflectance measurement can be perfectly compensated for silvered materials (both silvered-glass and polymer film reflectors) when the reflectometer is recalibrated using a reference mirror of the same material type, but there is an increase in the measured value if no recalibrations are applied (because the instrument itself is affected by the temperature). Consequently, no corrections are needed for these silvered materials if the reflectometer is recalibrated when temperature variations occur. A frequent calibration is recommended (every $5{ }^{\circ} \mathrm{C}$ ) if relevant temperature changes occur between one measurement and another (i.e., outdoor measurements in large solar plants).

On the other hand, when the effect of the ambient temperature in the reflectometer response is balanced through frequent recalibrations (Fig. 11), the effect of the temperature on the measured reflectance of aluminum reflectors is clearly seen. This might be due to a different nature of the reference mirror used. This phenomenon is attenuated by the reflectometer behavior due to temperature, provoking the tendency observed in Fig. 10, when no recalibrations are applied. As frequent calibrations shall be done (to eliminate the influence of the temperature on the device), the following corrections should be applied to the reflectance measurement to obtain the correct value, $\rho_{\lambda, \varphi}$, for aluminum \#1 (5) and aluminum \#2 (6) samples:

$$
\begin{aligned}
\Delta \rho_{\lambda, \varphi} & =-0.0001 \cdot T+0.0006 ; R^{2}=0.9041 \\
\Delta \rho_{\lambda, \varphi} & =-0.0004 \cdot T+0.0001 ; R^{2}=0.9641 .
\end{aligned}
$$

As the correction equation depends on the aluminum type, it is advisable to perform a similar experiment for each aluminum reflector.

2) Instrument Unit Itself: The results of the test carried out with the five reflectometers are presented in Table IV, as the average and standard deviation of the ten reflectance measurements taken. As can be seen, the measurements taken with every individual reflectometer have a null standard
TABLE IV

Average and Standard Deviation Reflectance of the $4.0-\mathrm{mm}$

\begin{tabular}{|c|c|c|c|c|c|c|c|c|c|c|}
\hline \multirow{2}{*}{$\begin{array}{l}\text { Reflectometer } \\
\text { serial number }\end{array}$} & \multicolumn{2}{|c|}{1119} & \multicolumn{2}{|c|}{1117} & \multicolumn{2}{|c|}{1116} & \multicolumn{2}{|c|}{1110} & \multicolumn{2}{|c|}{060} \\
\hline & $\overline{\boldsymbol{X}}$ & $\sigma$ & $\overline{\boldsymbol{X}}$ & $\sigma$ & $\overline{\boldsymbol{X}}$ & $\sigma$ & $\overline{\boldsymbol{X}}$ & $\sigma$ & $\overline{\boldsymbol{X}}$ & $\sigma$ \\
\hline$\rho_{\lambda, \varphi}(-)$ & 0.960 & 0.000 & 0.960 & 0.000 & 0.962 & 0.000 & 0.959 & 0.000 & 0.958 & 0.000 \\
\hline
\end{tabular}
SILVERED-GLASS SAMPLE FOR EACH INSTRUMENT UNIT

TABLE V

Average and Standard Deviation Reflectance of the $4.0-\mathrm{mm}$ SilverED-GLASS SAMPLE FOR THE Two CALIBRATION MIRROR TYPES

\begin{tabular}{ccccc}
\hline \hline \multirow{2}{*}{ Calibration mirror } & \multicolumn{2}{c}{ 119 Silvered thin glass (internal) } & \multicolumn{2}{c}{ Silvered-glass 4 mm (external, ext4) } \\
& $\bar{X}$ & $\boldsymbol{\sigma}$ & $\overline{\boldsymbol{X}}$ & $\boldsymbol{\sigma}$ \\
\hline$\rho_{\lambda, \varphi}(-)$ & 0.960 & 0.000 & 0.960 & 0.000
\end{tabular}

deviation, which indicates a proper stability of each instrument itself. However, there are important differences when the five instrument units are compared among them (i.e., considering the 50 measurements done), thus obtaining results with a Gaussian distribution of a standard deviation of $\sigma=0.0014$. Hence, it is always recommended to use the same instrument when a set of measurements is taken, while an uncertainty of $u_{\text {reff,unit }}=0.0014$ can be considered when the reflectance values measured with different units are compared.

3) Reference Mirror: Table V includes the results of the test performed to study the influence of the calibration mirror type, thus indicating the average and standard deviation of the ten reflectance measurements. As can be observed, the standard deviation of the measurements is slightly higher when the reflectometer's own reference is used. This could be because normally the internal mirror undergoes higher degradation than the external one (because it cannot be recalibrated or replaced in the lab in the case of deterioration). Therefore, it is recommended to use an appropriate external reference mirror to do the calibration process of the reflectometer to have more stable results. If the results of this test are treated independently of the reference mirror used (i.e., considering the 20 values obtained), the standard deviation of the Gaussian distribution achieved is $\sigma=0.0010$. Consequently, an uncertainty of $u_{\text {refl, ref }}=0.0010$ can be considered when reflectance values measured with different calibration mirrors are compared.

Regarding practical issues, it is important to consider that the internal calibration mirrors are subjected to certain factors that might influence the calibration quality: soiling (sometimes difficult to eliminate), positioning instability, misalignment, etc. On the other hand, the use of an external calibration mirror might lead to inconveniencies for outdoor measurements. This external reference must be recalibrated periodically with a master standard and replaced in the case of deterioration.

4) Stability Over Time: The mean reflectance after $7 \mathrm{~h}$ is $\rho_{s, h}=0.961$ and its $\sigma=0.073$. Data follow a triangular distribution, and consequently, the uncertainty associated with this parameter is calculated from the variance of this probability distribution by applying the following equation:

$$
u_{\text {refl,time }}^{2}=\frac{a^{2}}{6}
$$


TABLE VI

AVerage and Standard Deviation Reflectance of the 4.0-mm Silvered-Glass SAMPLE FOR Ambient Light TeST

\begin{tabular}{cccccc}
\hline \hline \multirow{2}{*}{ Ambient light } & & With & & \multicolumn{2}{c}{ Without } \\
& $\bar{X}$ & & $\sigma$ & $\bar{X}$ & $\sigma$ \\
\hline$\rho_{\lambda, \varphi}(-)$ & 0.961 & & 0.000 & 0.961 & 0.000 \\
\hline
\end{tabular}

TABLE VII

Average and Standard Deviation Reflectance of All Samples FOR DifFERENT $\varphi$. N.A.: NOT APPLICABLE

\begin{tabular}{|c|c|c|c|c|c|c|c|}
\hline \multirow{2}{*}{$\varphi(\operatorname{mrad})$} & \multicolumn{2}{|c|}{223.0} & \multicolumn{2}{|c|}{$\overline{12.5}$} & \multicolumn{2}{|c|}{$\overline{7.5}$} & \multirow[b]{2}{*}{$u_{\mathrm{refl}, \varphi}$} \\
\hline & $\overline{\boldsymbol{X}}$ & $\sigma$ & $\overline{\boldsymbol{X}}$ & $\sigma$ & $\overline{\boldsymbol{X}}$ & $\sigma$ & \\
\hline Silvered-glass $0.95 \mathrm{~mm}$ & 0.960 & 0.000 & 0.960 & 0.000 & 0.960 & 0.000 & 0.000 \\
\hline Silvered-glass $2.1 \mathrm{~mm}$ & 0.961 & 0.000 & 0.961 & 0.000 & 0.959 & 0.0007 & 0.0011 \\
\hline Silvered-glass $4.0 \mathrm{~mm}$ & 0.962 & 0.000 & 0.961 & 0.000 & 0.962 & 0.0005 & 0.0005 \\
\hline Silvered polymer film\#1 & 0.941 & 0.0003 & 0.934 & 0.000 & 0.929 & 0.0005 & n.a. \\
\hline Silvered polymer film $\# 2$ & 0.965 & 0.0004 & 0.955 & 0.0004 & 0.913 & 0.007 & n.a. \\
\hline Aluminum\#1 & 0.857 & 0.0005 & 0.837 & 0.0008 & 0.812 & 0.0005 & n.a. \\
\hline Aluminum $\# 2$ & 0.797 & 0.0009 & 0.777 & 0.0009 & 0.741 & 0.0008 & n.a. \\
\hline
\end{tabular}

where $a$ is the half of the triangle base. As in this case, $a=0.001$ and $u_{\text {reff,time }}=0.0004$. From a practical point of view, this means that this uncertainty should be added when the instrument is being used during a full working day (without any recalibration).

5) Ambient Light: Table VI shows the results of the test carried out to check the influence on the ambient light in the reflectometer. In this case, no changes were detected in the measured reflectance values, and, as a consequence, the standard deviation is null and $u_{\text {reff,light }}=0.000$. From a practical point of view, this involves that the measurements can be taken with ambient light without any problem.

6) Acceptance Angle: In this case, all samples were included because it was observed that materials with lower specularity are more affected by changes in $\varphi$. As shown in Table VII, the reflectance measurements of the three silvered-glass samples present standard deviations null at $\varphi=\{12.5,23.0\} \mathrm{mrad}$ and negligible at $\varphi=7.5 \mathrm{mrad}$, which indicates a good homogeneity for every $\varphi$. Also, the average reflectance is quite similar for different $\varphi$, thus informing that the scattering in this type of reflectors is very low. As a consequence, these results confirm that silvered-glass reflectors are highly specular and they can even be measured with the independence of the $\varphi$ (as it was already suggested in [11]). If this is the case, the uncertainty to be considered, $u_{\text {refl, } \varphi}$, was derived from the standard deviation of the normal distributions (i.e., the series of 30 data for each sample type), as it is indicated in the last column of Table VII. Regarding the silvered-polymer films, the behavior of the two samples analyzed is quite different. On the one hand, standard deviations of each $\varphi$ are much lower for polymer \#1 than those for polymer \#2. In addition, the differences in the average reflectance from one $\varphi$ to another are higher in polymer \#2, thus indicating a higher scattering for this sample. Hence, it is demonstrated that the scattering in this type of mirrors
TABLE VIII

AVERAGE AND Standard DeViation Reflectance of Three SAMPLES WITH DIFFERENT FRONT-LAYER THICKNESS FOR TEN POSITIONS OF THE SCREW

\begin{tabular}{cccccccc}
\hline \hline \multirow{2}{*}{ Reflector sample } & \multicolumn{2}{c}{ Aluminum\#1 } & \multicolumn{2}{c}{ Silvered-glass $0.95 \mathbf{~ m m}$} & \multicolumn{2}{c}{ Silvered-glass 4.0 mm } \\
& $\overline{\boldsymbol{X}}$ & $\boldsymbol{\sigma}$ & $\overline{\boldsymbol{X}}$ & $\boldsymbol{\sigma}$ & $\overline{\boldsymbol{X}}$ & $\sigma$ \\
\hline$\rho_{\lambda, \varphi}(-)$ & 0.837 & 0.0007 & 0.960 & 0.000 & 0.961 & 0.000 \\
\hline \hline
\end{tabular}

depends on the polymer layer deposited onto the silver layer and the degradation status. Finally, both aluminum mirrors present similar standard deviation for the three $\varphi$, with higher values than the rest of the materials, as well as significant differences between average reflectance values, thus indicating greater scattering in aluminum reflectors than that in silvered ones. In general, the discrepancies among the values obtained at different $\varphi$ for silvered-polymer film and aluminum samples point out that this parameter must be properly selected to measure these kinds of reflectors. Therefore, the calculation of $u_{\mathrm{refl}, \varphi}$ makes no sense for aluminum and polymer films.

7) Central Screw Position: The results of the study about the influence of the central support are shown in Table VIII. As observed, null variations were noticed regarding mirror thickness and the number of central screw turns in the two silvered-glass samples. In the case of the aluminum sample, a normal distribution with a very low standard deviation ( $\sigma=0.0007$ ) was obtained. If this $\sigma$ is compared to the corresponding one in Table VI (alumnium \#1 sample at $\varphi=12.5 \mathrm{mrad}$, ten repetitions with the central screw fixed), a similar value is achieved, which indicates that the modification of the position of the central screw is not affecting the results. Hence, the screw position showed a neglected influence on the reflectance, and it is considered that this factor does not contribute to the type-B uncertainty of the device $\left(u_{\text {ref,scr }}=0.000\right)$. From a practical point of view, it is safe to always maintain the screw in one specific position and adjust the optical beam only with the two outer screws.

8) Operator: Table IX presents the results of the test performed to study the influence of the operator experience. As can be seen, the standard deviation of the measurements is slightly higher for a little experienced operator (mainly in nonglass mirrors). A more experienced operator reaches more homogeneous results, and consequently, it is highly recommended that the operator that is going to use the reflectometer receives proper training to assure stable values. In addition, it is important that all the measurements belonging to the same test campaign are done by the same operator. Finally, if reflectance measurements are compared for each sample type and regardless of the operator's level of expertise (i.e., considering the 30 values for each sample), Gaussian distributions are detected in all samples, and the uncertainty to be considered is presented in the last column of Table IX.

9) Curvature of the Mirrors: The results obtained for this experiment are presented in Table $\mathrm{X}$, where the mean and the standard deviation of the specular reflectance values are depicted for both flat and curved statuses of the mirror samples. In the silvered-glass mirror, no influence of the curvature is detected, as the specularity is very high. However, 
TABLE IX

Total Standard DeViation AMONG Three DifFERENT Operators

\begin{tabular}{cccccccc}
\hline \hline Operator's level of & \multicolumn{2}{c}{ Expert } & \multicolumn{2}{c}{ Medium } & \multicolumn{2}{c}{ Novel } & uref,ope \\
expertise & $\overline{\boldsymbol{X}}$ & $\boldsymbol{\sigma}$ & $\overline{\boldsymbol{X}}$ & $\boldsymbol{\sigma}$ & $\overline{\boldsymbol{X}}$ & $\boldsymbol{\sigma}$ & \\
\hline Silvered-glass 0.95 mm & 0.960 & 0.000 & 0.959 & 0.0005 & 0.961 & 0.0004 & 0.0010 \\
Silvered-glass 2.1 mm & 0.961 & 0.000 & 0.961 & 0.000 & 0.962 & 0.000 & 0.0005 \\
Silvered-glass 4.0 mm & 0.961 & 0.000 & 0.959 & 0.000 & 0.959 & 0.0005 & 0.0009 \\
Silvered polymer film\#1 & 0.934 & 0.000 & 0.933 & 0.0005 & 0.933 & 0.0005 & 0.0005 \\
Silvered polymer film\#2 & 0.952 & 0.0003 & 0.952 & 0.0004 & 0.952 & 0.007 & 0.0006 \\
Aluminum\#1 & 0.836 & 0.0005 & 0.837 & 0.0008 & 0.836 & 0.0012 & 0.0009 \\
Aluminum\#2 & 0.777 & 0.0009 & 0.773 & 0.0009 & 0.776 & 0.0015 & 0.0019 \\
\hline \hline
\end{tabular}

TABLE X

Average and Standard Deviation ReFlectance of THREe MirRor SAMPLES With DiFFERENT CURVATURES, MEASURED With THE REFLECTOMETER

\begin{tabular}{cccccc}
\hline \hline \multirow{2}{*}{ Reflector sample } & \multicolumn{2}{c}{ Flat shape } & \multicolumn{2}{c}{ Curved shape } & \\
& $\overline{\boldsymbol{X}}$ & $\boldsymbol{\sigma}$ & $\overline{\boldsymbol{X}}$ & $\boldsymbol{\sigma}$ & $\boldsymbol{u}_{\text {refl,curv }}$ \\
& & & & & \\
\hline Silvered-glass $0.95 \mathrm{~mm}$ & 0.959 & 0.000 & 0.959 & 0.000 & 0.000 \\
Silvered polymer film\#1 & 0.931 & 0.000 & 0.931 & 0.0007 & 0.0006 \\
Aluminum\#2 & 0.785 & 0.0003 & 0.783 & 0.0005 & 0.001 \\
\hline \hline
\end{tabular}

for silvered-polymer film \#1 and aluminum \#2, if the flatand curved-shape measurements are compared, the value of $u_{\text {reff,curv }}$ obtained is 0.0006 and 0.001 , respectively. The reason behind this influence is that the equipment is not able to compensate for the curvature of the mirror sample when it presents a certain scattering. Thus, if several specular reflectance measurements are taken in several positions of a concentrator with different curvatures, an uncertainty should be added in materials with scattering, such as aluminum or polymers.

10) Combined Type-B Uncertainty of the Reflectometer:

Combined type-B uncertainty of the reflectometer, $u_{\mathrm{B} \text {,refl }}$, is calculated with the information presented in Sections IIIA1-III-A9 and also given in Tables I and III, by applying (3). Considering the data given by the manufacturer (Table I), the corresponding uncertainties are $u_{\text {refl,acc }}=0.002$ and $u_{\text {reff,res }}=0.0006$ since the resolution follows a rectangular distribution. According to Table III, the uncertainty to be considered for the calibration is $u_{\text {refl,cal }}=0.0015$ if the external reference mirror used is OMT-216035-01 or OMT-214044-02 (manufactured by OMT), while the value is $u_{\text {refl, ref }}=0.0013$ for the reference PAV-D-2 (manufactured by NRC). Consequently, the minimum type-B uncertainty for the reflectometer is obtained by (8) for the OMT references and (9) for the NRC reference

$$
\begin{aligned}
u_{\mathrm{B}, \mathrm{refl}} & =\sqrt{u_{\mathrm{refl}, \mathrm{acc}}^{2}+u_{\mathrm{refl}, \mathrm{res}}^{2}+u_{\mathrm{refl}, \mathrm{cal}}^{2}} \\
& ==\sqrt{0.002^{2}+0.0006^{2}+0.0015^{2}}=0.003 \\
u_{\mathrm{B}, \mathrm{refl}} & =\sqrt{u_{\mathrm{refl}, \mathrm{acc}}^{2}+u_{\mathrm{reff,res}}^{2}+u_{\mathrm{refl}, \mathrm{cal}}^{2}} \\
& =\sqrt{0.002^{2}+0.0006^{2}+0.0015^{2}}=0.003 .
\end{aligned}
$$

TABLE XI

INDIVIDUAL AND COMBINED UNCERTAINTIES OF THE REFLECTOMETER FOR DIFFERENT REFLECTORS

\begin{tabular}{ccccccc}
\hline \hline Uncertainty & $\boldsymbol{u}_{\text {refl,unit }}$ & $\boldsymbol{u}_{\text {ref,ref }}$ & $\boldsymbol{u}_{\text {ref,,ime }}$ & $\boldsymbol{u}_{\text {ref, } \boldsymbol{\varphi}}$ & $\boldsymbol{u}_{\text {ref,,ope }}$ & $\boldsymbol{u}_{\text {ref,,curv }}$ \\
\hline Silvered-glass $0.95 \mathrm{~mm}$ & 0.0014 & 0.0010 & 0.0004 & 0.000 & 0.0010 & 0.000 \\
Silvered-glass $2.1 \mathrm{~mm}$ & 0.0014 & 0.0010 & 0.0004 & 0.0011 & 0.0005 & n.a. \\
Silvered-glass $4.0 \mathrm{~mm}$ & 0.0014 & 0.0010 & 0.0004 & 0.0005 & 0.0009 & n.a. \\
Silvered polymer film\#1 & 0.0014 & 0.0010 & 0.0004 & n.a. & 0.0005 & 0.0006 \\
Silvered polymer film\#2 & 0.0014 & 0.0010 & 0.0004 & n.a. & 0.0006 & n.a. \\
Aluminum\#1 & 0.0014 & 0.0010 & 0.0004 & n.a. & 0.0009 & n.a. \\
Aluminum\#2 & 0.0014 & 0.0010 & 0.0004 & n.a. & 0.0019 & 0.001 \\
\hline \hline
\end{tabular}

If a coverage factor of $k=2$ is considered, the minimum expanded uncertainty obtained for the reflectometer is $U_{\mathrm{B}, \text { refl }}=$ 0.006 for all material samples. With respect to the other parameters studied, the ambient light and the position of the central screw did not show any impact on the results, giving null uncertainties, $u_{\text {reff,light }}=u_{\text {refl,screw }}=0.000$. In the case of the influence of ambient temperature, no uncertainty or correlation should be added for silvered reflectors when recalibrations are done every time that temperature changes around $5 \mathrm{C}$, while a correction should be applied to the reflectance results for aluminum mirrors (see Section III-A1). Finally, Table XI presents the uncertainties that must be combined with the values presented in (8) or (9) for different samples when the rest of the parameters analyzed are involved in the measurement process.

Finally, if all different individual uncertainties are considered and included in (3), the value obtained is $u_{\mathrm{B} \text {, refl }}=0.003$ for all materials, except for aluminum \#2, whose uncertainty is $u_{\mathrm{B}, \text { refl }}=0.004$. This means that in general, the uncertainty is quite independent of the material (for those typically used in CST applications) and the main source of uncertainty is the accuracy of the instrument reported by the manufacturer. If a coverage factor of $k=2$ is considered, the maximum expanded uncertainty calculated for the reflectometer is $U_{\mathrm{B}, \text { refl }}=0.006$ for all material samples, except for aluminum \#2, whose expanded uncertainty is $U_{\mathrm{B}, \mathrm{refl}}=0.008$.

\section{B. Type-B Uncertainties of the Spectrophotometer}

This section includes the results of the tests described in Section II-F to calculate the type-B uncertainties of the spectrophotometer.

1) Ambient Temperature: According to the results obtained (see Table XII), the differences in the reflectance values measured at the two ambient temperatures studied, $T=[16,26]{ }^{\circ} \mathrm{C}$, were null for all materials. Consequently, this device is very stable in the range of operating temperatures suitable for an air-conditioned laboratory, and neither correction nor uncertainty must be considered in relation to this parameter $\left(u_{\text {spec,tem }}=0.000\right)$ if laboratory standard ambient conditions are assured.

2) Reference Mirror: Table XIII shows the reflectance and standard deviation of the ten reflectance repetitions done, 
TABLE XII

Average and Standard Deviation for Two Different LaboraTORY AMBIENT TEMPERATURES

\begin{tabular}{ccccc}
\hline \hline \multirow{2}{*}{ Ambient temperature $\left({ }^{\circ} \mathbf{C}\right)$} & \multicolumn{2}{c}{$\mathbf{1 6}$} & \multicolumn{2}{c}{$\mathbf{2 6}$} \\
& $\overline{\boldsymbol{X}}$ & $\boldsymbol{\sigma}$ & $\overline{\boldsymbol{X}}$ & $\boldsymbol{\sigma}$ \\
\hline Silvered-glass $0.95 \mathrm{~mm}$ & 0.950 & 0.000 & 0.950 & 0.000 \\
Silvered-glass $2.1 \mathrm{~mm}$ & 0.950 & 0.000 & 0.950 & 0.000 \\
Silvered-glass $4.0 \mathrm{~mm}$ & 0.947 & 0.000 & 0.947 & 0.000 \\
Silvered polymer film\#1 & 0.922 & 0.000 & 0.922 & 0.000 \\
Silvered polymer film\#2 & 0.934 & 0.000 & 0.934 & 0.000 \\
Aluminum\#1 & 0.897 & 0.000 & 0.897 & 0.000 \\
Aluminum\#2 & 0.870 & 0.000 & 0.871 & 0.000 \\
\hline \hline
\end{tabular}

TABLE XIII

Average and Standard Deviation Reflectance of Measurement With THREE REFERENCE MiRRORS

\begin{tabular}{|c|c|c|c|c|c|c|}
\hline \multirow{3}{*}{ Reflector material } & \multicolumn{6}{|c|}{ External reference mirrors } \\
\hline & \multicolumn{2}{|c|}{ ext4 } & \multicolumn{2}{|c|}{ ext2 } & \multicolumn{2}{|c|}{ extAl } \\
\hline & $\bar{X}$ & $\sigma$ & $\bar{X}$ & $\sigma$ & $\bar{X}$ & $\sigma$ \\
\hline Silvered-glass $4.0 \mathrm{~mm}, \rho_{s, h}(-)$ & 0.947 & 0.000 & 0.948 & 0.000 & 0.951 & 0.002 \\
\hline Aluminum $\# 1, \rho_{s, h}(-)$ & 0.897 & 0.000 & 0.898 & 0.000 & 0.900 & 0.001 \\
\hline
\end{tabular}

thus measuring the 4-mm silvered-glass sample and the aluminum\#1 sample using the three different reference mirrors (see Table III).

As appreciated in Table XIII, the standard deviations of the measured reflectance of silvered-glass and aluminum samples are null when the two silvered-glass references are used (ext2 and ext4). However, the standard deviation of the measurements when the aluminum reference mirror is used is higher in both cases. Hence, it is advisable to work with the silvered-glass reference mirror (independently of the glass thickness). If no distinctions between reference mirrors are done when the spectrophotometer measurements are performed, a maximum uncertainty of $u_{\text {spec,ref }}=0.002$ and $u_{\text {spec,ref }}=0.001$ can be obtained for silvered-glass and aluminum samples, respectively.

3) Stability Over Time: The mean reflectance and its standard deviation of the 280 measurements performed in this test were $\rho_{s, h}=0.947$ and 0.000 , respectively. This means that a great stability over time was shown by the spectrophotometer and no uncertainty is derived from this parameter, that is, $u_{\text {spec,time }}=0.000$.

4) Ambient Light: Fig. 12 shows the results of the test performed to check the effect of the ambient light in the spectrophotometer. This graph represents the standard deviation of the ten repetitions performed both with and without the opaque cover, as a function of the wavelength. As can be seen, slightly smaller standard deviation values were achieved when the cover was utilized (blue curve), thus indicating a higher stability of the measurements in this case.

For an easier comparison, Table XIV shows the results of the effect of the cover as the average and standard deviation values of $\rho_{s, h}$. As can be observed, null variations were

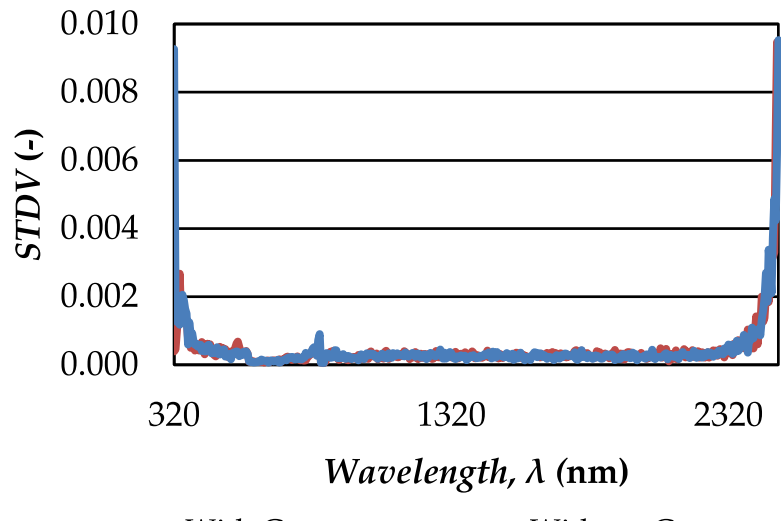

Fig. 12. Standard deviation spectra of the ten repetitions for the measurements with and without an opaque cover.

TABLE XIV

Average and Standard Deviation Reflectance of Measurement WITH AND WITHOUT COVERS

\begin{tabular}{cccccc}
\hline Use of cover & \multicolumn{3}{c}{ With } & \multicolumn{3}{c}{ Without } \\
& $\overline{\boldsymbol{X}}$ & & $\boldsymbol{\sigma}$ & $\overline{\boldsymbol{X}}$ & $\boldsymbol{\sigma}$ \\
\hline$\rho_{\lambda, h}(-)$ & 0.947 & & 0.000 & 0.947 & 0.000 \\
\hline \hline
\end{tabular}

detected in $\rho_{s, h}$ when the cover was employed, while slight influence was suffered without the cover $(\sigma=0.0005)$. Consequently, it is recommended to use a cover to avoid any possible influence of the ambient light in the reflectance results. If results are compared independently of the use of this kind of protection (i.e., considering the 20 reflectance values), a Gaussian distribution is obtained, which gives an uncertainty of $u_{\text {spec,light }}=0.0006$.

5) Detector Response Time: Fig. 13 shows the standard deviation of the ten repetitions at three detector response times (the minimum, the maximum, and a mix of them) as a function of the wavelength. The mixed curve represents the result of the measurement done at the maximum response time in the whole solar wavelength range, except in $\lambda=[600,880]$ $\mathrm{nm}$, where the minimum response time was selected. As appreciated, the higher standard deviation was obtained at the fastest response time (0.04 s) compared to the slowest detector response time (1 s), being the mixed curved intermediate among them.

Table XV shows the results of the $\rho_{s, h}$ for the three response times, as well as the time consumed in each case. As the detector response time should be a compromise between the accuracy and the testing time, the mix solution is recommended. If no attention is paid to the detector response time (i.e., the 30 measurements are considered), the maximum uncertainty associated is $u_{\text {spec,det }}=0.0004$.

6) Curvature of the Mirrors: Regarding the $u_{\text {refl,curv }}$, as represented in Table XVI, the three types of mirrors did not show any differences between hemispherical reflectance when the surface is flat or curved because in this case, the effect of the scattering provoked by the aluminum and polymer is palliated. 


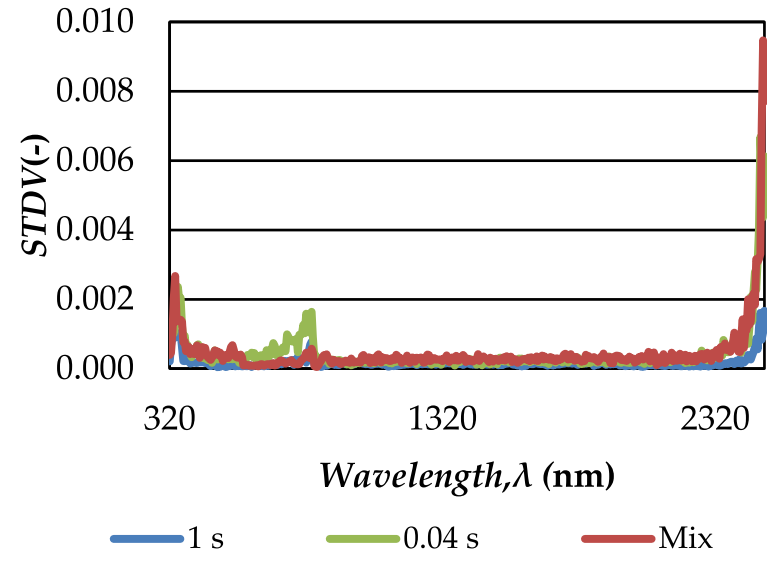

Fig. 13. Standard deviation spectra of the ten repetitions for the measurements at the minimum and maximum detector response time.

TABLE XV

Average and Standard Deviation Reflectance of Measurement at Three Different Detector Response Times, As Well as the TESTING TIME EMPLOYED

\begin{tabular}{|c|c|c|c|c|c|c|}
\hline \multirow{2}{*}{$\begin{array}{c}\text { Detector response } \\
\text { time (s) }\end{array}$} & \multicolumn{2}{|c|}{0.04} & \multicolumn{2}{|c|}{ Mix } & \multicolumn{2}{|c|}{1} \\
\hline & $\bar{X}$ & $\sigma$ & $\bar{X}$ & $\sigma$ & $\bar{X}$ & $\sigma$ \\
\hline$\rho_{s, h}(-)$ & 0.947 & 0.0008 & 0.947 & 0.0007 & 0.961 & 0.000 \\
\hline Time (s) & \multicolumn{2}{|c|}{150} & \multicolumn{2}{|c|}{214} & \multicolumn{2}{|c|}{610} \\
\hline
\end{tabular}

7) Combined Type-B Uncertainty of the Spectrophotometer: Combined type-B uncertainty of the spectrophotometer, $u_{\mathrm{B}, \mathrm{spec}}$, is calculated with the information presented in the previous sections and also given in Tables I and III, by applying (4). Considering data given by the manufacturer (Table I), the corresponding uncertainty due to the instrument accuracy is $u_{\text {spec,acc }}=0.007$. Although the resolution of the spectrophotometer is higher than that of the reflectometer to avoid confusion when data from the two instruments are provided, it is a common practice to consider the same number of decimals for both $\rho_{\lambda, \varphi}$ and $\rho_{s, h}$. This means that $u_{\text {spec,res }}=0.001$. According to Table III, the uncertainty to be considered is $u_{\text {spec,ref }}=0.0015$ if the external reference mirror used is OMT-216035-01 or OMT-214044-02 (manufactured by OMT), while for the reference PAV-D-2 (manufactured by NRC), the value is $u_{\text {refl, ref }}=0.0013$. Consequently, the minimum type-B uncertainty for the spectrophotometer is calculated by (10) for the OMT references and (11) for the NRC reference

$$
\begin{aligned}
u_{\mathrm{B}, \mathrm{spec}} & =\sqrt{u_{\mathrm{spec}, \mathrm{acc}}^{2}+u_{\mathrm{spec}, \mathrm{res}}^{2}+u_{\mathrm{spec}, \mathrm{cal}}^{2}} \\
& =\sqrt{0.007^{2}+0.001^{2}+0.0015^{2}}=0.007 \\
u_{\mathrm{B}, \mathrm{spec}} & =\sqrt{u_{\mathrm{spec}, \mathrm{acc}}^{2}+u_{\mathrm{spec}, \mathrm{res}}^{2}+u_{\mathrm{spec}, \mathrm{cal}}^{2}} \\
& =\sqrt{0.007^{2}+0.001^{2}+0.0013^{2}}=0.007 .
\end{aligned}
$$

If a coverage factor of $k=2$ is considered, the minimum expanded uncertainty obtained for the spectrophotometer is $U_{\mathrm{B}, \mathrm{spec}}=0.014$ for all material samples. With respect to the other parameters studied, the ambient temperature and the stability over time did not show any impact on the results, thus giving null uncertainty,
TABLE XVI

Average and Standard DeViation ReFlectance of Three Samples With a DifFERENT CURVATURE MEASURED IN THE SPECTROPHOTOMETER

\begin{tabular}{cccccc}
\hline \hline \multirow{2}{*}{ Reflector sample } & \multicolumn{2}{c}{ Flat } & \multicolumn{2}{c}{ Curve } & \\
& $\overline{\boldsymbol{X}}$ & $\boldsymbol{\sigma}$ & $\overline{\boldsymbol{X}}$ & $\boldsymbol{\sigma}$ & $\boldsymbol{u}_{\text {refl,curv }}$ \\
& & & & & \\
\hline Silvered-glass $0.95 \mathrm{~mm}$ & 0.950 & 0.000 & 0.950 & 0.000 & 0.000 \\
Silvered polymer film\#1 & 0.920 & 0.000 & 0.920 & 0.000 & 0.000 \\
Aluminum\#2 & 0.861 & 0.000 & 0.861 & 0.000 & 0.000 \\
\hline \hline
\end{tabular}

TABLE XVII

INDIVIDUAL AND COMBINED UNCERTAINTIES OF THE SPECTROPHOTOMETER FOR DIFFERENT TYPES OF SOLAR REFLECTORS

\begin{tabular}{ccccc}
\hline \hline Uncertainty & $\boldsymbol{u}_{\text {spec,unit }}[7]$ & $\boldsymbol{u}_{\text {spec,ref }}$ & $\boldsymbol{u}_{\text {spec,light }}$ & $\boldsymbol{u}_{\text {spec,det }}$ \\
\hline Silvered-glass reflectors & 0.002 & 0.002 & 0.0006 & 0.0004 \\
Aluminum reflectors & 0.002 & 0.001 & 0.0006 & 0.0004 \\
\hline \hline
\end{tabular}

$u_{\text {spec,tem }}=u_{\text {spec,time }}=u_{\text {spec,curv }}=0.000$. Table XVII presents the uncertainties to be combined with the values calculated in (10) or (11) when the rest of the parameters are involved.

Finally, if all different individual uncertainties are considered and included in (4), the value obtained is $u_{\mathrm{B}, \mathrm{spec}}=0.008$ for all materials. This means that in general, the uncertainty is independent of the material (for those typically used in CST applications) and the main source of the uncertainty is the accuracy of the device, reported by the manufacturer. If a coverage factor of $k=2$ is considered, $U_{\mathrm{B} \text {,spec }}=0.016$ for all material samples.

\section{DISCUSSION}

The following specific remarks from the tests performed with the reflectometer might be highlighted.

1) The combined type-B uncertainty is $u_{\mathrm{B}, \mathrm{refl}}=0.003$ if the only factors considered are the accuracy of the instrument, its resolution, and the reference mirror used to calibrate the device.

2) Ambient temperature changes do not influence the reflectance measurement process of silvered-glass mirrors when intermediate recalibrations are carried out. However, corrections are needed for aluminum materials when temperature changes exist between measurements. Hence, a frequent calibration is recommended if relevant ambient temperature fluctuations occur.

3) Although the influence of the instrument unit is not critical (with $u_{\text {reff, unit }}=0.00014$ ), it is recommended to use the same instrument when a set of measurements is going to be taken.

4) It is advisable to use an appropriate external reference mirror to calibrate the reflectometer (instead of its own calibration mirror) because higher measurement stability is obtained. Moreover, internal references are more prone to deteriorate. If both types of reference mirrors are used indistinctly, the uncertainty to be added is $u_{\text {refl, ref }}=0.0010$. 
5) If no recalibrations are carried out over the testing time, the contribution of this factor on the type-B uncertainty should also be considered, that is, $u_{\text {refl,time }}=0.0004$.

6) It is demonstrated that the influence of the external light in this device is null ( $\left.u_{\text {refl, light }}=0.000\right)$.

7) Specular reflectance of silvered-glass reflectors is not highly affected by $\varphi$ due to the high specularity $u_{\text {refl, } \varphi}<$ 0.0011. However, the reflectance values obtained for silvered polymer film and aluminum reflector samples significantly fluctuate depending on $\varphi$. Therefore, it is recommended to measure nonglass reflectors with the appropriate $\varphi$ (depending on the technology).

8) It is safe to keep the central screw always in one specific position $\left(u_{\text {refl, screw }}=0.000\right)$.

9) The more experienced the operator is, the more homogeneous the results are. The experience of the operator has only little influence on the accuracy of the result if he or she is trained on the correct procedure before measuring. The uncertainty in this case depends on the material type.

10) An uncertainty should be added in materials with scattering, such as aluminum or polymers, when a concentrator with different curvatures is measured, being $u_{\text {refl,curv }}=0.001$ for the aluminum and $u_{\text {refl,curv }}=$ 0.0006 for the polymer.

11) Finally, if all the parameters studied are considered in the type-B uncertainty calculation, $u_{\mathrm{B}, \text { refl }}=0.003$ for all the reflectors considered in this study except for the aluminum\#2 whose uncertainty is $u_{\mathrm{B} \text {, refl }}=0.004$.

In addition, the main results obtained from the tests performed with the spectrophotometer are given as follows.

1) The combined type-B uncertainty is $u_{\mathrm{B} \text {,spec }}=0.007$ if the only factors considered are the accuracy of the device, its resolution, and the reference mirror.

2) In the range of ambient temperatures from $16{ }^{\circ} \mathrm{C}$ to $26{ }^{\circ} \mathrm{C}$, the spectrophotometer does not suffer reflectance changes due to the temperature $\left(u_{\text {spec,tem }}=0.000\right)$.

3) Using the same measurement method but different units of the spectrophotometer gives a high reproducibility, that is, $u_{\text {spec,unit }}=0.002$.

4) Silvered-glass mirror references are the most adequate alternative to take the measurements because the uncertainty added to the measure is lower than that in aluminum reference. If samples are measured indistinctly with both types of references, $u_{\text {spec,tem }}=0.002$ should be considered for silvered-glass samples and $u_{\text {spec,tem }}=$ 0.001 for aluminum samples.

5) Regarding the stability over time, it has been evidenced that the equipment is really stable if laboratory ambient conditions are constant $\left(u_{\text {spec,time }}=0.000\right)$.

6) The influence of the ambient light is a parameter to take into account because an excessive illumination in the laboratory might affect the measurement. If measurements are carried out in bright rooms, $u_{\text {spec,light }}=0.0006$ should be added.

7) Detector response time affects the spectrophotometer measurements. The differences of standard deviation between the faster and slower detector response times are quite insignificant along the whole solar spectrum, except in the range $\lambda=[600,880] \mathrm{nm}$, where the slower method obtains much better results. Thus, a deal between standard deviation and time of measurement should be achieved. If no attention is paid to the detector response time for this specific device, the maximum uncertainty associated is $u_{\mathrm{spec} \text {,det }}=0.0004$. But for other types of spectrophotometer, the contribution of this factor to the uncertainty could be more relevant.

8) The influence of the curvature, $u_{\text {refl,curv }}$, does not affect the uncertainty in the spectrophotometer measurements.

9) When all the influences are considered in the uncertainty calculation, the maximum uncertainty of $u_{\mathrm{B} \text {, spec }}=0.008$ is obtained, regardless of the type of sample.

\section{CONCLUSiON}

This article demonstrates that both the reflectometer and the spectrophotometer are adequate devices for optical measurements of reflector materials for CST technologies. Two specific commercial devices were used to perform this study. The maximum expanded type-B uncertainty calculated is $U_{\mathrm{B}, \text { refl }}=0.006$ for monochromatic specular reflectance and $U_{\mathrm{B}, \mathrm{spec}}=0.016$ for solar-weighted hemispherical reflectance. Moreover, it is recommended to recalibrate the reflectometer regularly when ambient temperature fluctuations exist between measurements to use the same equipment and the same reference mirror to calibrate the device in all the measurement processes and properly train the operators before using the device. Regarding the spectrophotometer, it is advisable to employ different detector response times along the spectrum in order to obtain a suitable measurement for the sake of both the time invested and the accuracy. In addition, silveredglass reference mirrors shall be used to measure silvered as well as aluminum reflector specimens because of their lower uncertainty compared to aluminum references.

\section{ACKNOWLEDGMENT}

The authors would like to thank A. M. G. Leitón from CIEMAT, Almería, Spain, for her valuable comments.

\section{REFERENCES}

[1] K. H. Solangi, M. R. Islam, R. Saidur, N. A. Rahim, and H. Fayaz, "A review on global solar energy policy," Renew. Sustain. Energy Rev, vol. 15, no. 4, pp. 2149-2163, May 2011.

[2] P. G. V. Sampaio and M. O. A. González, "Photovoltaic solar energy: Conceptual framework," Renew. Sustain. Energy Rev., vol. 74, pp. 590-601, Jul. 2017.

[3] H. Price et al., "Advances in parabolic trough solar power technology," J. Sol. Energy Eng., vol. 124, no. 2, pp. 109-125, Apr. 2002.

[4] D. Mills, "Advances in solar thermal electricity technology," Sol. Energy, vol. 76, nos. 1-3, pp. 19-31, Jan. 2004.

[5] A. Fernández-García, E. Zarza, L. Valenzuela, and M. Pérez, "Parabolictrough solar collectors and their applications," Renew. Sustain. Energy Rev., vol. 14, no. 7, pp. 1695-1721, 2010.

[6] M. Montecchi et al., "Hemispherical reflectance results of the SolarPACES reflectance round robin," Energy Procedia, vol. 69, pp. 1904-1910, May 2015.

[7] S. Meyen, A. Fernández-García, C. Kennedy, and E. Lüpfert, "Standardization of solar mirror reflectance measurements-Round robin test," in Proc. SolarPACES Int. Conf., Perpignan, France, 2010, pp. 1-8. 
[8] R. Gee, R. Brost, Z. Guangdong, and G. Jorgensen, "An improved method for characterizing reflector specularity for parabolic trough concentrators," in Proc. SolarPACES Int. Conf., Perpignan, France, 2010, pp. $1-8$.

[9] A. Heimsath, G. Kutscheidt, and P. Nitz, "Measuring reflectanceoverview and influence on optical performance," in Proc. SolarPACES Int. Conf., Granada, Spain, 2011, pp. 1-8.

[10] M. Montecchi, "Approximated method for modelling hemispherical reflectance and evaluating near-specular reflectance of CSP mirrors," Sol. Energy, vol. 92, pp. 280-287, Jun. 2013.

[11] A. Fernández-García et al., "Simplified analysis of solar-weighted specular reflectance for mirrors with high specularity," in Proc. AIP Conf. SolarPACES, Cape Town, South Africa, 2016, Art. no. 130006.

[12] F. Sutter, S. Meyen, P. Heller, and R. Pitz-Paal, "Development of a spatially resolved reflectometer to monitor corrosion of solar reflectors," Opt. Mater, vol. 35, no. 8, pp. 1600-1608, Jun. 2013.

[13] A. Heimsath, T. Schmid, and P. Nitz, "Angle resolved specular reflectance measured with VLABS," Energy Procedia, vol. 69, pp. 1895-1903, May 2015.

[14] F. Sutter, S. Meyen, A. Fernández-García, and P. Heller, "Spectral characterization of specular reflectance of solar mirrors," Sol. Energy Mater. Sol. Cells, vol. 145, pp. 248-254, Feb. 2016.

[15] F. Sutter, A. Fernández-García, A. Heimsath, M. Montecchi, and C. Pelayo, "Advanced measurement techniques to characterize the nearspecular reflectance of solar mirrors," in Proc. SOLARPACES, Int. Conf. Concentrating Sol. Power Chem. Energy Syst., Casablanca, Morocco, 2019, Art. no. 110003.

[16] M. Montecchi, "Solar mirror qualification setup, the key instrument in a new strategy for evaluating off-normal near-specular solar-reflectance," Rev. Sci. Instrum., vol. 89, no. 12, Dec. 2018, Art. no. 123114

[17] A. Fernández-García et al., "Parameters and method to evaluate reflectance properties of reflector materials for concentrating solar power technology," Oficial Reflectance Guideline SolarPACES version 3.0, Almería, Spain, Tech. Rep., Mar. 2018.

[18] Reflector Panels for Concentrating Solar Technologies, Standard UNE 206016, 2018

[19] A. Fernández-García, F. Sutter, L. Martínez-Arcos, C. Sansom, F. Wolfertstetter, and C. Delord, "Equipment and methods for measuring reflectance of concentrating solar reflector materials," Sol. Energy Mater. Sol. Cells, vol. 167, pp. 28-52, Aug. 2017.

[20] Solar Thermal Electric Plants-Part 1-1: Terminology, document IEC TS 62862-1-1, 2018.

[21] A. Fernández-García, F. Sutter, J. Fernández-Reche, and E. Lüpfert, The Performance of Concentrated Solar Power (CSP) Systems: Analysis, Measurement and Assessment, 1st ed., Amsterdam, The Netherlands: Elsevier, 2017, ch. 3, pp. 67-98.

[22] Glass in Building-Determination of Light Transmittance, Solar Direct Transmittance, Total Solar Energy Transmittance, Ultraviolet Transmittance and Related Glazing Factors, Standard ISO 9050, 2003.

[23] R. B. Pettit, "Characterizing solar mirror materials using portable reflectometers," Sandia Nat. Lab., Albuquerque, NM, USA, Tech. Rep. SAND82-1714, 1982.

[24] Portable Specular Reflectometer Model 15R-USB Operation and Maintenance Manual, Devices Services, Dallas, TX, USA, 2009.

[25] Portable Specular Reflectometer Model 15R-USB Operating Instructions, Devices Services, Dallas, TX, USA, 2009.

[26] J. L. Taylor, Reflectance Measurements of Materials Used in Solar Industry. Shelton, CT, USA: PerkinElmer, 2009.

[27] High Performance Lambda Spectrophotometers Hardware Guide, PerkinElmer, Beaconsfield, U.K., 2007.

[28] C. Sansom, A. Fernández-García, P. King, F. Sutter, and A. G. Segura, "Reflectometer comparison for assessment of back-silvered glass solar mirrors," Sol. Energy, vol. 155, pp. 496-505, Oct. 2017.

[29] S. Bouaddi, A. Ihlal, and A. Fernández-García, "Comparative analysis of soiling of CSP mirror materials in arid zones," Renew. Energy, vol. 101, pp. 437-449, Feb. 2017.

[30] F. Sutter, J. Wette, F. Wiesinger, A. Fernández-García, S. Ziegler, and R. Dasbach, "Lifetime prediction of aluminum solar mirrors by correlating accelerated aging and outdoor exposure experiments," Sol. Energy, vol. 174, pp. 149-163, Nov. 2018.

[31] F. Wiesinger et al., "Assessment of the erosion risk of sandstorms on solar energy technology at two sites in Morocco," Sol. Energy, vol. 162, pp. 217-228, Mar. 2018.
[32] A. García-Segura, A. Fernández-García, M. J. Ariza, F. Sutter, and L. Valenzuela, "Degradation of concentrating solar thermal reflectors in acid rain atmospheres," Sol. Energy Mater. Sol. Cells, vol. 186, pp. 92-104, Nov. 2018.

[33] C. Sansom, A. Fernández-García, F. Sutter, H. Almond, P. King, and L. Martínez-Arcos, "Soiling and cleaning of polymer film solar reflectors," Energies, vol. 9, no. 12, p. 1006, Nov. 2016.

[34] A. García-Segura, A. Fernández-García, M. J. Ariza, F. Sutter, and L. Valenzuela, "Effects of reduced sulphur atmospheres on reflector materials for concentrating solar thermal applications," Corrosion Sci. vol. 133 , pp. $78-93$, Apr. 2018

[35] A. Fernández-García, A. Juaidi, F. Sutter, L. Martínez-Arcos, and F. Manzano-Agugliaro, "Solar reflector materials degradation due to the sand deposited on the backside protective paints," Energies, vol. 11, no. 4 , p. 808 , Mar. 2018

[36] A. García-Segura, A. Fernández-García, M. J. Ariza, F. Sutter, and L. Valenzuela, "Durability studies of solar reflectors: A review," Renew. Sustain. Energy Rev., vol. 62, pp. 453-467, Sep. 2016.

[37] J. Wette, F. Sutter, A. Fernández-García, S. Ziegler, and R. Dasbach, "Comparison of degradation on aluminum reflectors for solar collectors due to outdoor exposure and accelerated aging," Energies, vol. 9, no. 11, p. 916, Nov. 2016.

[38] Evaluation of Measurements Data-Guide to the Expression of Uncertainty in Measurement, document JCGM 100, 2008.

[39] J. Howell, M. P. Mengüc, and R. Siegel, Thermal Radiation Heat Transfer, 6th ed. Boca Raton, FL, USA: CRC Press, 2015.

[40] Radiometric and Photometric Properties of Materials; Methods of Measurement for Photometric and Spectral Radiometric Characteristics Factors Part 3, document DIN 5036, 1979.

[41] Standard Test Method for Solar Absorptance, Reflectance, and Transmittance of Materials Using Integrating Spheres, Standard ASTM E 903-96, 2005.

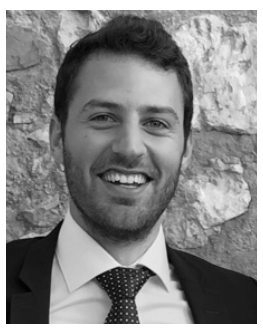

Francisco Buendía-Martínez was born in Larva, Spain, in 1994. He received the degree in chemistry from the University of Córdoba, Córdoba, Spain, in 2016, the master's degree in energies and fuels for the future from the University Autónoma of Madrid, Madrid, Spain, in 2017. He is currently pursuing the Ph.D. degree with CIEMAT. His Ph.D. thesis is on "Lifetime estimation of materials for solar reflectors" where the aim is to provide correlations which predict the solar mirror lifetime.

$\mathrm{He}$ has participated in several scientific articles and he actively collaborates in the RAISELIFE Project as a Scientist (Gran Agreement Nr. 686008), carrying out durability tests in primary and secondary mirrors.

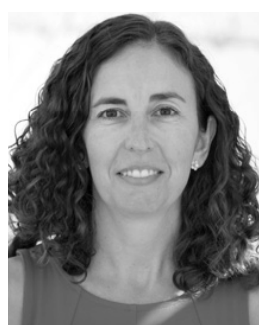

Aránzazu Fernández-García was born in Madrid, Spain, in 1977. She received the B.S./M.Eng. degree in industrial engineering from the University of Malaga, Málaga, Spain, in 2004, and the M.S. degree in solar energy and the Ph.D. degree in environmental engineering from the University of Almería, Almería, Spain, in 2007 and 2013, respectively. She has developed her research activity in CIEMAT-PSA, Almería, since 2002. She is the author of two books and three book chapters. She is the coauthor of 34 publications in international journals, 78 contributions to different international conferences and symposiums and more than 300 technical reports. She has attended 16 international conferences. She has supervised three Ph.D. students, six master's students, and 12 undergraduate students. She has been involved in $18 \mathrm{EU}$ and four Spanish founded Research and development grants and has been scientific responsible of 35 Research and development cooperation agreements with industries. She is currently the Site Manager of the laboratories for optical characterization and durability testing of solar reflectors at the PSA (OPAC). Her research activities are mainly focused on the optical measurement and durability testing of solar reflectors (both in outdoor conditions and accelerated aging) and the optimization of the cleaning methods for concentrating solar power plants. 


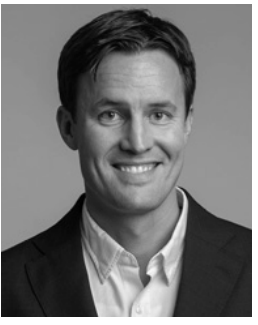

Florian Sutter was born in Stuttgart, Germany, in 1983. He received the Diploma degree in mechanical engineering from the Karlsruhe Institute of Technology, Karlsruhe, Germany, in 2008. He is currently pursuing the Ph.D. degree in solar research engineering with RWTH Aachen University, Aachen, Germany, which is concerned with the measurement and modeling of the optical durability of enhanced aluminum reflectors.

Since 2012, he has been a Team Leader of the "Durability of Components" Group, Department of Qualification, DLR Institute of Solar Research, Almería, Spain. He manages the German Team, OPAC Laboratory, which is jointly operated with CIEMAT at the Plataforma Solar de Almería, Almería. He has participated in various industry and research projects as a Scientist and a Project Leader. He currently acts as a Coordinator of the EU H2020 Project RAISELIFE (Grant Agreement Nr. 686008), which is concerned with the durability testing of key materials for concentrating solar power.

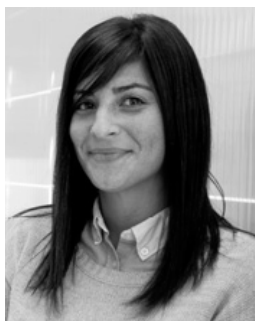

Lucía Martínez-Arcos was born in Almería, Spain, in 1989. She received the HNC Diploma degree from Higher Technician in Analysis and Quality Control Laboratory, Los Ángeles Training Institute, Almería, in 2009, and the HNC Diploma degree from Higher Technician in Environmental Chemistry Laboratory, Los Ángeles Training Institute, in 2011.

In 2011, she worked as a Quality Control Technician with the cement industry. In 2011, she joined Ciemat-PSA, Almería, as a Research Laboratory Technician.

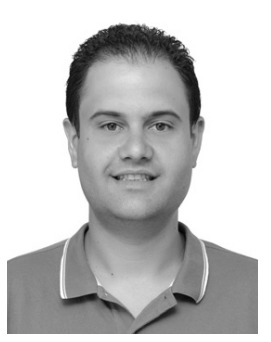

Tomás Jesús Reche-Navarro was born in Almería, Spain, in 1986. He received the VET Diploma degree in laboratory expert from the Los Angelesn Training Institute, Almería, in 2007, the HNC Diploma degree from Higher Technician in Analysis and Quality Control Laboratory, Los Ángeles Training Institute, in 2009, and the HNC Diploma degree in advanced expert in prevention of occupational risks from the Los Ángeles Training Institute, in 2011.

From 2008 to 2013, he worked as a Quality Control Technician at cement industry. In 2013, he joined the German Aerospace Center (DLR), Cologne, Germany, as a Research Laboratory Technician.

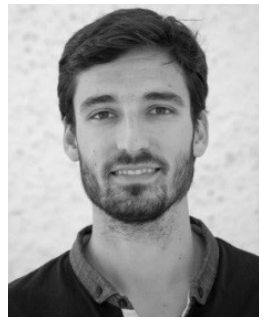

Alejandro García-Segura was born in Spain, in July 14, 1988. He received the Chemical Engineering degree and the Ph.D. degree in applied environmental sciences from the University of Almería, Almería, Spain, in 2012 and 2018, respectively. His $\mathrm{Ph} . \mathrm{D}$. thesis was focused on the durability analyses of reflectors used in concentrating solar thermal systems under the effect of corrosive gases typically found near polluted environments.

He is currently a Project Manager of the Multidisciplinary Research Center in Basque Country.

His research interests include renewable energies, CSP systems, accelerated aging tests, optical properties analysis, and corrosion assessment.

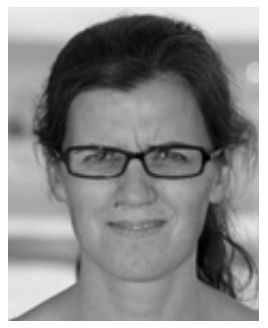

Loreto Valenzuela received the degree in physics and the degree in electronics engineering from the University of Granada, Granada, Spain, in 1994 and 1996, respectively, and the Ph.D. degree from the University of Almería, Almería, Spain, in 2007.

In 1997, she joined Plataforma Solar de Almerían (CIEMAT-PSA), Almería, where she started to work in the process control of parabolic-troughs solar collectors systems. Since then, she has acquired a great deal of experience in the design, modeling, simulation, development of testing procedures and test benches, and experimental evaluation of complete prototypes and components for concentrating solar thermal technology, mainly related to line-focus solar collectors. She is currently the Head of the Research Unit of Solar Concentrating Systems, CIEMAT-PSA. She has been involved in 28 Research and development projects and more than 30 bilateral Research and development contracts with industries or public entities. She is the coauthor of 89 journal articles or conference proceedings.

Dr. Valenzuela is a member of the Spanish and international standardization committees defining standards for solar thermal power plants and their components (AENOR/CTN206/SC117 and IEC TC117). She has been leading the sub-program "Line Focusing Systems" of the joint program on Concentrating Solar Power of the European Energy Research Alliance (EERA) since 2014. 